\title{
Interaction between the supercontinent cycle and the evolution of intrinsically dense provinces in the deep mantle
}

\author{
Sean J. Trim, Julian P. Lowman \\ Version Post-print/accepted manuscript \\ Citation Trim, S. J., and J. P. Lowman (2016), Interaction between the \\ (published version) supercontinent cycle and the evolution of intrinsically dense provinces \\ in the deep mantle, J. Geophys. Res. Solid Earth, 121, 8941- \\ 8969, doi:10.1002/2016JB013285.
}

How to cite TSpace items

Always cite the published version, so the author(s) will receive recognition through services that track citation counts, e.g. Scopus. If you need to cite the page number of the author manuscript from TSpace because you cannot access the published version, then cite the TSpace version in addition to the published version using the permanent URI (handle) found on the record page.

This article was made openly accessible by $U$ of $T$ Faculty.

Please tell us how this access benefits you. Your story matters. 


\title{
Interaction between the supercontinent cycle and the evolution of intrinsically dense provinces in the deep mantle
}

\author{
Sean J. Trim ${ }^{1}$, Julian P. Lowman ${ }^{1,2}$ \\ ${ }^{1}$ Department of Physics, University of Toronto, Toronto, Ontario, Canada \\ ${ }^{2}$ Also at Department of Physical and Environmental Sciences, University of Toronto Scarborough, Toronto, Ontario, \\ Canada

\section{Key Points:} \\ - Time-dependence and supercontinent assembly frequency is increased by the presence \\ of dense provinces in the lower mantle \\ - Anomalously hot upper mantle consistently appears above the margins of composition- \\ ally dense provinces \\ - Generally one or two dense lower mantle provinces are present and intermittently ex- \\ hibit periods of fixity
}

Corresponding author: S. J. Trim, strimephysics.utoronto.ca 


\section{Abstract}

Shear-wave travel times in the Earth's deep mantle reveal broad steep-sided seismologically distinct provinces lying on the Core-Mantle Boundary (CMB). The longevity and permanence of the two large principal provinces, located below the sites of present-day Africa and the Pacific Ocean, have become a matter of great interest. Examination of the flood basalt record and kimberlite eruption dating suggests the presence of these provinces may disclose a deep mantle component with a compositionally distinct origin that plays a role in the generation of mantle plumes at preferred locations. By extension, the presence of these provinces may affect the supercontinent cycle. Implementing a mantle convection model featuring distinct continental lithosphere and a Compositionally Anomalous and Intrinsically Dense (CAID) component, we study the distribution and mobility of naturally forming compositionally distinct provinces and their impact on model supercontinent assembly. We employ 2D Cartesian geometry calculations of thermo-chemical convection with extremely low compositional diffusion to model Earth-like convective vigor on a global scale and find that an intrinsically dense mantle component generally aggregates into one or two broad provinces. The positions of the provinces are time-dependent but in many of our calculations the province locations are characterized by periods of fixity that reach several hundred million years. Eras of province and associated plume fixity are punctuated by periods of relatively rapid migration. A correlation between supercontinent position and the locations of CAID provinces is not supported by our findings. However, we find the frequency of supercontinent assemblies increases when CAID provinces are present.

\section{Introduction}

Various lines of evidence suggest that Earth's $\mathrm{D}^{\prime \prime}$ region is as rich in chemical [Trampert et al., 2004; Šrámek et al., 2013], phase [Murakami et al., 2004; Wookey et al., 2005] and structural heterogeneity [Tackley, 2012] as its lithosphere. In particular, seismic imaging of the lower mantle [Karato and Karki, 2001; Garnero and McNamara, 2008] indicates a rich diversity in properties that opens multiple questions regarding the existence of travel time anomalies and anisotropy [Ni et al., 2002; Long and Becker, 2010] and the nature of their origin [Davies et al., 2015]. (However, tomography is strongly limited in its ability to infer evolution of the deep mantle or the origin of potentially deeply sourced phenomena like hotspots.)

In contrast to the deeper mantle that drives its motion, continual change of the buoyancy distribution in the lithosphere is more clearly documented; due to the breadth, endurance and 
accessibility of the geologic record [Condie, 2002]. Fossil histories [Wilson, 1966] and paleomagnetism [e.g., Runcorn, 2013; Mitchell et al., 2014] reveal how inherently buoyant continental cratons drift across the Earth's surface, both responding to and influencing the migration and evolution of plate boundaries. A well documented corollary of continental drift is the supercontinent cycle; characterized by the alternating assembly and dispersal of the majority of the Earth's continental cratons. Modeling studies show that supercontinents influence the mantle's thermal evolution due to their influence on subduction zone positioning, and suggest feedback between continental drift and the deep mantle [e.g., Gurnis, 1988; Lowman and Gable, 1999; Li and Zhong, 2009; O'Neill et al., 2009; Heron and Lowman, 2011; Lenardic et al., 2011; Rolf et al., 2012; Heron et al., 2015]

Encompassed within and extending above the $\mathrm{D}^{\prime \prime}$ region beneath Africa and the Pacific are the Large Low Shear-wave Velocity Provinces (LLSVPs). The LLSVPs have been inferred to be the seismic signature of broadly distributed compositionally anomalous material with a chemistry that is distinct from the ambient mantle [e.g., Masters et al., 2000; Garnero and McNamara, 2008; Lekic et al., 2012]. They are defined by atypical velocity magnitude anomalies, sharp lateral gradients in shear wave velocity at their margins and anti-correlated shear wave and bulk sound velocities, all of which have received attention for their support in building a case hypothesizing that LLSVPs are compositional in origin [Ritsema et al., 1998; Ni et al., 2002; To et al., 2005; Karato and Karki, 2001; Saltzer et al., 2001; Brodholt et al., 2007]. However, a chemical origin for LLSVPs is not a settled issue [e.g., Davies et al., 2015]. Several studies [Schuberth et al., 2009, 2012; Davies et al., 2015] have shown that the velocity anomalies and gradients that are consistent with a compositional origin for the LLSVPs are also obtainable in isochemical mantle circulation models. Nevertheless, a compositional origin for LLSVPs does remain a viable premise, particularly supported by evidence of an anti-correlation between density and shear wave velocity anomalies [e.g., Ishii and Tromp, 1999, 2004; Trampert et al., 2004].

Given their location at the base of the mantle, if LLSVPs are compositionally distinct then they are both Compositionally Anomalous and Intrinsically Dense (for brevity we adopt the acronym CAID to describe material with these properties) relative to the ambient mantle. Potential origins for CAID material in the deep mantle include a primordial source, preserved in relatively sequestered assemblages [e.g., Allègre et al., 1987; Boyet and Carlson, 2006; Labrosse et al., 2007; Jackson et al., 2010; Deschamps et al., 2011], chemical interaction with the outer core [Humayun et al., 2004] and segregation of oceanic slab material [Tackley et al., 2005; Bran- 
denburg et al., 2008] (however mantle convection modeling by Li and McNamara [2013] suggests this is difficult to achieve).

The LLSVPs may have occupied their current positions overlying the Core-Mantle Boundary (CMB) for a period reaching back to Pangea's assembly and possibly even further into the past [Dziewonski et al., 2010]. By reconstructing the eruption site history of Large Igneous Provinces (LIPs) over the past $200 \mathrm{Myr}$, Torsvik et al. [2006] concluded that most LIPs were formed by plumes originating at the present-day edges of the LLSVPs. Additionally, Burke et al. [2008] suggest that sharp lateral temperature gradients associated with the steep sides of the LLSVPs lead to Plume Generation Zones (PGZs) that generate LIPs. However, in terms of serving as preferred locations for plume originating LIPs, recent studies [Austermann et al., 2014] call into question any significant statistical difference between the margins of LLSVPs and the interiors of slower than average shear wave velocity regions. (Although, a correlation between slower than average shear wave velocity regions and LIP sites is supported by statistical analysis [Austermann et al., 2014].) Consequently, the connection between LLSVPs and plume generation remains tenable, but previously implemented indicators for assessing the fixity of LLSVP margins may not be robust.

If the LLSVPs are chemically distinct complexes, it is possible that their locations and morphology may be influenced by the dynamic evolution of the mantle [e.g., Zhang et al., 2010] just as the low density cratons of the continents are driven to drift across the globe by the mantle's evolution. If there is any analogue with continental drift, the nature of this motion and continuity of these complexes could evolve with some sort of cyclicity, with eras of aggregation and almost negligible motion interrupted by periods of significant velocity and dispersal. Furthermore, if not only periods of supercontinent assembly but also the amalgamation of CAID provinces both occur in cycles, then some feedback between these phenomena could exist.

In this study we examine the mutual interaction between continents and CAID provinces using two-dimensional thermochemical numerical models of mantle convection featuring dynamically evolving tectonic plates and deeply deposited compositionally dense material. Previous studies showed that the range of possible density contrasts for compositional provinces, relative to the ambient mantle, is limited to a confined band [e.g., Tackley, 2012; Trim et al., 2014]. For greater densities, the CAID component settles into a contiguous layer [e.g., Le Bars and Davaille, 2002, 2004a; Styles et al., 2011] that envelops the core. For lower density contrasts, the dense component mixes into the mantle so that the provinces rapidly decrease in volume or even become completely entrained within time periods less than a supercontinent 
cycle. In this study we specify a density contrast for the CAID component of our calculations that is conducive to forming distinct provinces over periods of a billion years of model time. (However, it is not our intent to model Earth history. Instead, we aim to observe long-term model behavior with a given set of parameters, rather than a brief temporal sample that may be of atypical character.) We do not consider a source for the CAID material that can add to or replenish the initial volume of the dense component. In addition, we do not consider distinct viscous properties for the CAID material. (However, we note that a distinct viscosity would likely shift the density contrast band that allows the formation of provinces [Li et al., 2014]. Moreover, entrainment of the dense component does result over the time scales modeled in our calculations [McNamara and Zhong, 2004a].) A 2D model is implemented in order to specify parameters consistent with terrestrial mantle convection while resolving thermochemical dynamics over periods of billions of years.

Previous findings [McNamara and Zhong, 2004a; Tan et al., 2011; Trim et al., 2014; Li et al., 2014] have shown that intrinsically dense material can mix with the ambient mantle, settle into a contiguous layer, coalesce into multiple widely dispersed piles or networks of ridges, or form a small number of large provinces. We explore whether stable broad compositional provinces can be formed in a vigorously convecting system featuring evolving plate motion and model continents, and a possible correlation between supercontinent and CAID province positions. A primary interest is in possible feedback between CAID provinces and continent dispersement. In particular, we examine the frequency of supercontinent cycles and formation, the viability of forming plume generation zones and the distribution and mobility of compositional provinces.

\section{Method}

\subsection{Governing equations}

Mantle convection is studied using a Boussinesq infinite Prandtl number fluid in a 2D Cartesian geometry. The system features two distinct mantle and lithosphere components. The mantle is comprised of ambient silicates and CAID material. The lithosphere features inherently buoyant conducting continents and oceanic material that participates in mantle convection. Thermochemical convection is modeled by solving the non-dimensional equations for composition, mass, momentum and energy conservation: 


$$
\frac{\partial C}{\partial t}=-\boldsymbol{v} \cdot \nabla C,
$$

and

$$
\boldsymbol{\nabla} P-\boldsymbol{\nabla} \cdot(2 \eta \dot{\boldsymbol{\epsilon}})=\left(R a_{T} T-R a_{C} C\right) \hat{\boldsymbol{z}},
$$

respectively [e.g., van Keken et al., 1997; Montague and Kellogg, 2000; McNamara and Zhong, 2004a; Deschamps and Tackley, 2009], where $\boldsymbol{v}$ is the velocity, $P$ is the pressure, $\eta$ is the dynamic viscosity, $\dot{\boldsymbol{\epsilon}}$ is the deviatoric strain-rate tensor, $T$ is the superadiabatic temperature, $C$ is the composition, $\hat{z}$ is a unit vector antiparallel to gravity, and $t$ is time. The thermal and compositional Rayleigh numbers based on the viscosity at the surface are

$$
R a_{T}=\frac{\rho_{0} g \alpha \Delta T d^{3}}{\kappa \eta_{\text {surf }}}
$$

and

$$
R a_{C}=\frac{\Delta \rho_{C} g d^{3}}{\kappa \eta_{\text {surf }}},
$$

where $\rho_{0}$ is a reference density, $g$ is the acceleration due to gravity, $\alpha$ is the thermal expansivity, $\Delta T$ is the superadiabatic temperature difference across the mantle, $d$ is the system thickness, $\kappa$ is the thermal diffusivity, $\eta_{\text {surf }}$ is the dynamic viscosity at the surface, and $\Delta \rho_{C}$ is the compositional density contrast between the enriched and ambient mantle materials.

By implementing the Boussinesq approximation we neglect shear heating and adiabatic temperature changes. The importance of both scale as the system depth, $d$, over the temperature scale-height $\left(H_{T}=C_{P} / g \alpha\right.$, where $C_{P}$ is the specific heat at constant pressure). The Boussinesq approximation assumes that $d / H_{T}<<1$, however, for whole mantle convection 
this ratio is approximately 0.5. Jarvis and McKenzie [1980] employed the anelastic-liquid equations, that include the effects of viscous dissipation and variable adiabatic temperature gradients, and studied the effect of increasing $d / H_{T}$ from values satisfying the Boussinesq requirement to a ratio in excess of that describing whole mantle convection. They found that for a mantle-like ratio, only small details in the flow structure of the convection were present and conclude viscous stress fields can be neglected when constructing thermal models of the Earth. Accordingly, we adopt the Boussinesq approximation here due to the simplification of the conservation equations it allows, particularly for modeling thermo-chemical convection [e.g., van Keken et al., 1997; Tackley and King, 2003; McNamara and Zhong, 2004a, 2005; Samuel and Evonuk, 2010]

Our calculations do not include modeling of the endothermic phase change between the upper and lower mantle, however, we do model a sharp increase in viscosity at $660 \mathrm{~km}$ depth that has been inferred to accompany the phase change. Previous studies have found that with a Rayleigh number appropriate for modeling whole mantle convection, slab sinking rates are dominated by viscosity stratification in comparison to the influence of phase changes [Shahnas and Jarvis, 2007].

To solve the non-dimensional set of equations (1)-(4) we specify that the surface and basal temperatures are fixed at 0 and 1 , respectively. The depth and time-dependent non-dimensional viscosity is given by

$$
\eta=\Delta \eta_{P}(z) \Delta \eta_{T}^{-\bar{T}}=\Delta \eta_{P}(z) \exp (-\gamma \bar{T}),
$$

where $\Delta \eta_{T}$ and $\Delta \eta_{P}$ give the magnitude of viscosity variation due to the laterally-averaged temperature $\bar{T}(z, t)$ (i.e., the geotherm) and an intrinsic depth dependence (where $z=0$ and 1 at the base and surface of the system, respectively). The expansion of the temperature-dependence at the right-hand-side of equation (7) emphasizes that our formulation is equivalent to a FrankKamenetskii formulation using a laterally averaged temperature with a reference value of zero (the surface) corresponding to a nondimensional viscosity of one and $\gamma=\ln \left(\Delta \eta_{T}\right)$. In models featuring plate motion obtained through the implementation of plastic yielding, it has been shown that the use of a geotherm-dependent viscosity profile results in similar surface mobility and heat flux to that found using a fully temperature-dependent viscosity [Stein and Hansen, 2014]. To model the inferred viscosity increase present in Earth's lower mantle [e.g., Hager, 1984; King and Masters, 1992; Mitrovica and Forte, 1997; Paulson et al., 2007], in this study 
we set $\Delta \eta_{P}=1$ for $z>0.77$ and $\Delta \eta_{P}=30$ for $z \leq 0.77$ (i.e., an increase by a factor of 30 below a depth of $660 \mathrm{~km}$ ). In addition, we set $\Delta \eta_{T}=10^{6}$ (a sufficient viscosity contrast for strong plates). Viscosity is not composition dependent. The calculation domain is periodic and has a free-slip base (surface velocity boundary conditions are discussed below).

In all calculations, we set $R a_{T}=10^{4}$ and take $d=2.9 \times 10^{6} \mathrm{~m}, \kappa=1.0 \times 10^{-6}$ $\mathrm{m}^{2} \cdot \mathrm{s}^{-1}, g=9.8 \mathrm{~m} \cdot \mathrm{s}^{-2}, \rho_{0}=3.3 \times 10^{3} \mathrm{~kg} \cdot \mathrm{m}^{-3}, \alpha=1.2 \times 10^{-5} \mathrm{~K}^{-1}, \Delta T=2700 \mathrm{~K}$, and $\eta_{\text {surf }}=2.6 \times 10^{24} \mathrm{~Pa} \cdot \mathrm{s}$. The volume-averaged thermal Rayleigh number, computed a posteriori using the globally averaged viscosity in place of $\eta_{\text {surf }}$ in equation (5), is approximately $10^{7}$ in all results discussed.

For thermochemical convection, the Boussinesq equation of state is

$$
\rho=\rho_{0}[1+\alpha \Delta T(B C-T)],
$$

where $B=R a_{C} / R a_{T}$ is the buoyancy ratio, equivalent to $\Delta \rho_{C} /\left(\rho_{0} \alpha \Delta T\right)$. Accordingly, $\Delta \rho_{C}=B \rho_{0} \alpha \Delta T$, so that $\Delta \rho_{C}=74.9 \mathrm{~kg} \cdot \mathrm{m}^{-3}$ for $B=0.7$, the buoyancy ratio chosen in this study (discussed below).

Equation (1) is solved using the tracer ratio method [Tackley and King, 2003]. Lagrangian tracer particles are used to track each chemical component. The tracers are advected using a second order Runge-Kutta method. The velocities are calculated at the tracer positions using a second order interpolation method. MC3D has been tested against the thermochemical benchmark described in van Keken et al. [1997] and produces results comparable to those therein in addition to those of more recent studies [e.g., Tackley and King, 2003; Samuel and Evonuk, 2010]. To track composition we use $2.56 \times 10^{7}$ tracers.

\subsection{Modeling plates and continents}

Equations (2) to (4) are solved using the code MC3D, utilizing a hybrid spectral finite difference scheme flow solver and a finite volume scheme for the solution of the energy equation [e.g., Gable et al., 1991; Koglin et al., 2005]. MC3D is second order accurate in time and space. Plates are dynamically modeled using a force-balance method that specifies time-dependent surface velocity boundary conditions. When utilizing the force-balance method, the surface velocities are piecewise spatially uniform over the broad areas separated by plate boundaries and are continuously updated to ensure that, at all times, the integrated shear stress calculated at the base of each plate is zero. Plates are incorporated in the solution domain and have a thick- 
ness that is time-dependent, does not vary laterally and is determined by the temperature defining the base of the plates. At this depth, the horizontally-averaged temperature is equal to a lithosphere transition temperature (assigned a non-dimensional value of 0.35 , a superadiabatic temperature that is $945 \mathrm{~K}$ hotter than the surface). Implementing the force-balance method, the surface velocities neither drive nor resist the convective flow [Gable et al., 1991; Brandenburg and van Keken, 2007]. All buoyancy related forces that drive plate motion, including slab sinking and thermal boundary layer (lithospheric) thickening [Hager and O'Connell, 1981] are incorporated into the force-balance calculation driving the plates, as are tractions arising from the motion of all plates. The latter tractions arise from a Stokes-flow generated circulation throughout the mantle, due to the motion of each plate [e.g., Davies, 1977; Gable et al., 1991].

Stresses at the plate boundaries are mitigated by implicit weak zones at the mobile plate boundaries [e.g., Koglin et al., 2005] defined by fixed widths over which velocities transition, approximately linearly, from zero to the plate velocity [Stein et al., 2014]. The width of these zones in our calculations is equal to the plate thickness and eliminates grid-scale velocity discontinuities at the plate boundaries where stress singularities would otherwise appear. The forcebalance method has been compared to rheological plate modeling methods in benchmark studies [e.g., Koglin et al., 2005; Stein et al., 2013] and shows very good agreement with the results of the rheological modeling methods in reproducing transitions between mobile and stagnantlid regimes [e.g., Stein et al., 2014]. In particular, the latter study found remarkable agreement of mean temperature, mobility (the ratio of surface to system root-mean-square velocities) and heat flux can be achieved by adjusting parameters such as the off-set viscosity (in the rheological plate modeling method) or the width of the weak zones (in the force-balance model). Mobility in both cases is determined by stresses acting on the model lithosphere and consequently agreement of this metric indicates that similar stress fields are reproduced from the equally similar temperature fields found with each modeling method.

In our calculations, lithosphere material is designated as either oceanic or continental. Plate boundaries move in response to thermal and compositional evolution and the nature of the adjacent lithosphere. Divergent boundaries move with the average velocity of neighboring plates and model symmetric seafloor spreading. Between adjacent oceanic plates convergent boundaries move with the velocity of the neighboring plate of youngest oceanic age. Thus, older denser plates are overridden and consumed at convergent margins. Convergent boundaries at continental margins move with the velocity of the continent so that the inherently buoyant continents are not subducted into the mantle. (Consequently, continental buoyancy is en- 
forced by tracking continental lithosphere stencils and the surface area covered by the continents remains fixed.)

A continental collision occurs at a convergent margin when all intervening oceanic material has been consumed. In this case, the adjacent cratons are sutured, eliminating the convergent margin. We find that plates must have a minimum size in order to preserve numerical stability. In all calculations, the minimum plate size is taken to be $400 \mathrm{~km}$ (or $1.4 \%$ of the total surface area). Plates that become smaller than this are split in two and sutured to the adjacent plates (modeling the absorption of "micro-plates" by large neighbors). The location of the split in the micro-plate is chosen to preserve the surface area ratio of the neighboring plates. For computational consistency, continents also have a minimum size of $400 \mathrm{~km}$.

New plate boundaries are created by plate rupturing where the vertically-averaged deviatoric horizontal normal stress exceeds a yield stress. Surface and end boundary normal and shear stresses on each plate are neglected, thus we determine deviatoric horizontal normal stresses in the plates by the integral of the shear tractions, $\tau_{x z}$, acting on the base of the plate. (This method is consistent with determination of the plate velocities by the force-balance calculation.) Specifically, if $h$ is the plate thickness, $x_{l}$ is the left position of a plate boundary and $x_{r}$ the right, a plate will break at a location $x_{p}$ where the nondimensional mean deviatoric horizontal normal stress

$$
\tilde{Y}=h^{-1} \int_{x_{l}}^{x_{p}} \tau_{x z} d x=h^{-1} \int_{x_{r}}^{x_{p}} \tau_{x z} d x
$$

exceeds the non-dimensional yield stress. (Note the integral of the shear tractions from $x_{l}$ to $x_{r}$ is zero). Oceanic and continental materials have distinct yield stress properties in our model. In oceans, the compressive yield stress is twice that of the tensile value. In continents, the tensile yield strength is less than or equal to the oceanic value. Compressive rupturing within continents is not modeled (i.e., orogenesis or continental block shortening). A dimensional yield stress value, $Y$, is defined as $\kappa \eta_{s u r f} \cdot d^{-2} \tilde{Y}$. Thus, a non-dimensional yield stress of 324 corresponds to a dimensional yield stress of $100 \mathrm{MPa}$.

\subsection{Calculation parameters and initial conditions}

To account for the distortion of the model geotherm in a plane-layer geometry versus a spherical shell, we omit internal heating from all calculations. Previous work by O'Farrell and Lowman [2010] and O'Farrell et al. [2013] has shown that obtaining geotherms correspond- 
ing to an Earth-like convective vigor, internal heating rate and core radius is achieved by the omission of internal heating in a plane-layer model. With this adjustment, the geotherms obtained in our calculations are similar to those that would be found in a spherical geometry with bimodal heating. Moreover, the thermal contrast between the model upwellings and geotherms is comparable to that which is found in a spherical shell.

Calculations feature aspect ratio 10 domains on a uniform grid with dimensions of $4000 \times 400$ cells. Further discussion of the implications of our choice of grid and tracer resolution is given in Appendix A. A high resolution is needed due to the large temperature gradients between the ambient mantle and subducting lithosphere (which effectively undergoes one-sided subduction because mass loss from the overriding plate cannot occur). An aspect ratio 10 domain has a width equal to the circumference of a great circle on a sphere defining a height of 1100 $\mathrm{km}$ above the core-mantle boundary. We employ a length scale close to mid-mantle depth to model a surface that allows for continent and plate dimensions comparable to Earth's while restricting amplification of the CMB dimensions.

All calculations are performed starting with identical initial temperature fields, plate boundary positions and surface age profiles. The initial temperature field was obtained in two steps. First, an isocompositional calculation with a free-slip surface was initialized with a perturbed conductive profile and then marched forward in time until reaching a statistically steady-state. Secondly, plates are introduced (i.e., we calculate piece-wise uniform surface velocities based on the stresses at the thermal lithosphere transition temperature) but with plate boundaries held in specified fixed positions. This stage continues while we wait for the thermal field to reach a statistically steady temperature. During this period uniform composition is specified and plates move between the fixed boundaries according to the force-balance criterion for calculating their velocities. A surface age profile is calculated by initially specifying a uniform age of zero and then calculating surface aging consistent with the evolving plate velocities. Surface age is set equal to zero when new lithosphere is formed at divergent margins. We calculate the increase in age with distance from divergent margins according to the history of each plate's velocity. The time required to reach a steady thermal field far exceeds the period required to calculate a surface age profile that is consistent with the past plate motion. Once the temperature has reached a steady mean we 1) allow for plate boundary motion and 2) introduce any compositionally enriched layer. In all calculations 1) and 2) are initiated at time $\mathrm{t}=0$, corresponding to the fields and surface age shown in Figure 1. 
Due to the symmetry of the plate sizes and the depth-dependent component of the viscosity, the initial thermal field we obtain is characterized by a pair of wide convection cells. Consequently, the initial condition features only one downwelling (model slab) and, due to a combination of the effect of the depth-dependent viscosity on upwelling dimensions in the lower mantle and the Cartesian geometry on the total number of lower boundary layer instabilities, a strong upwelling drawing neighboring broad hot thermals is also present. However, the geotherm in Fig. 1 indicates that the downwellings and upwellings have similar temperature contrasts to the background mantle as plumes and slabs in a bimodally heated spherical shell mantle. Once evolution of the boundaries is initiated the symmetry of the initial field is broken and multiple downwellings and plume clusters are frequently present.

In calculations featuring continents, three identical separate continents were added to the lithosphere at $t=0$. Each continent initially spans $10 \%$ of the surface for a total of $30 \%$ coverage, similar to continental lithosphere coverage on present-day Earth.

In calculations featuring compositional variations, we specify a buoyancy ratio of 0.7 for the compositional contrast. In previous work [Trim et al., 2014], it was found that a buoyancy ratio of 0.7 allows for long-lived compositional provinces while also maintaining surface mobility. For the Earth, if $2.5 \%$ of the volume of the spherical shell comprising the mantle envelops the outer core in a layer of uniform thickness, the layer's thickness would be $5 \%$ of the mantle depth. In our models, at $t=0$, a uniform-thickness layer of enriched material is introduced, comprising 5\% of the mantle depth and lying on the bottom boundary. We initialize our calculations with a CAID layer volume drawn from estimates of the upper range inferred for the present-day Earth (approximately 1.6-2.4\% of the mantle [Burke et al., 2008; Hernlund and Houser, 2008]). The volume of the compositional provinces in our calculations decreases over time due to mixing with the ambient mantle. Consequently, CAID volumes consistent with 1.6-2.4\% of the mantle volume exist for substantial periods during model evolution.

\section{Results}

The initial temperature, surface age and plate boundary locations for all models described in the remainder of this paper are shown in Figure 1. Also shown are the initial laterally averaged temperature as a function of depth and the corresponding non-dimensional viscosity as a function of depth. The increase in lower mantle viscosity at $660 \mathrm{~km}$ depth impedes pen- 
etration of the lower mantle by downwellings and reduces upwelling conduit width in the upper mantle.

The majority of the calculations feature compositional heterogeneity and/or continents. For those calculations, the initial composition field and continent positions are also shown in Fig. 1. All calculations are summarized in Table 1, using the naming syntax $\mathrm{ON} q_{-} \mathrm{CT} r\left\{{ }_{-} \mathrm{CH}\right\}$, where $q$ is the oceanic tensile yield strength in MPa, $r$ is the continental tensile yield strength in $\mathrm{MPa}$, and inclusion of the suffix _ $\mathrm{CH}$ indicates the presence of compositional heterogeneity. A single case is denoted with a superscript asterisk. This model features continents placed in different initial positions at time $t=0$ in comparison to the other cases (Initial Condition 2 in Fig. 1). For calculations that do not feature continents, $r$ has the value NA (i.e., not applicable). Table 1 also indicates the surface mobility regime for each model, the temporallyaveraged number of large compositional provinces, and the number of supercontinent assemblies, all of which are discussed below in detail. All calculations were integrated forward in time for four percent of a diffusion time $(\sim 10.6 \mathrm{Gyr})$. This integration time was selected to allow for an adjustment period following the initial condition while subsequently maintaining a sufficient time to observe multiple supercontinent cycles.

An example of model behavior is presented in Figure 2, which shows snapshots of temperature, composition, surface age, plate boundary and continent positions for Model ON100_CT80_CH at times of 5.0, 5.5, 6.0, 6.2 and 6.5 Gyr. This sequence illustrates the assembly and subsequent rupturing of a supercontinent. The compositional provinces are significantly hotter than ambient mantle temperatures and vigorous upwellings frequently form at their edges (discussed further below). At 5.0 Gyr there are three distinct continents that will subsequently aggregate as a result of plate boundary motion. Multiple compositional provinces exhibiting highly variable topography are dispersed over most of the base. At 5.5 Gyr a supercontinent assembly has occurred. The CAID piles that existed at 5.0 Gyr have been swept into a single province by the lateral motion of downwellings that have been consumed during a continental collision. At this time, a single compositional province has formed at the base of the suboceanic mantle. At $6.0 \mathrm{Gyr}$, a mantle flow reversal has occurred, as observed by plate boundaries transitioning from divergent to convergent and vice versa. Large upwellings are detached from a central compositional province. The positions of the supercontinent and compositional province continue to be anti-correlated. At $6.2 \mathrm{Gyr}$, the supercontinent has been ruptured above a vigorous upwelling not associated with the compositionally dense material. The diverging continental fragments migrate across the surface with velocities of approximately $10 \mathrm{~cm} / \mathrm{yr}$. A down- 
welling descending at the site of convergent plate motion has divided the wide compositional province into two distinct provinces [e.g., Zhang et al., 2010] with topography of a few hundred kilometers. At $6.5 \mathrm{Gyr}$, there are four continents and an increased number of plate boundaries. Several divergent boundaries (right side of panel) result from rupturing caused by a large upwelling at the edge of a compositional province. The two compositional provinces no longer occupy the same region of the lower mantle where they were found half a billion years earlier. Compared with the earlier snapshots (coinciding with supercontinent assembly), the surface age is relatively young due to new surface material forming at a large number of divergent plate boundaries.

Figure 3 shows several time series for Models ON80_CT80_CH (case a), ON100_CT80_CH (case b) and ON100_CT100_CH (case c). Time series of surface mobility (defined as the ratio between the surface and system root-mean-square velocities [e.g., Tackley, 2000]), plate count and continent count are shown in the left columns of cases a, b and c. Time series of the location of continents (gray) and basal coverage by the CAID provinces (red) are shown in the right column. In this figure, in order to appear in the plot, provinces are required to have a minimum width and composition: specifically $0.1 d(290 \mathrm{~km})$ and $0.3 \Delta \rho_{C}\left(22.5 \mathrm{~kg} \cdot \mathrm{m}^{-3}\right)$, respectively. Smaller and less dense anomalies at the base of the models are disregarded. In all these models, there is a supercontinent aggregation that occurs shortly after time $t=0.0$. In fact, similar model behavior is observed for the first $0.5 \mathrm{Gyr}$. This is expected since the differences in yield strengths in these calculations are only $25 \%$. However, over longer time scales major differences in the quantities presented evolve. In case a, a supercontinent forms five times, in case b seven times, and in case c four times (see Table 1). However, in case c supercontinent formation is almost continuous and continental dispersal is rare with the continent count fluctuating between one and two.

For Model ON80_CT80_CH, compositional provinces are situated both beneath and far from continents from approximately 0 to $4 \mathrm{Gyr}$. After $4 \mathrm{Gyr}$, the position of the provinces are anti-correlated with the position of continents for the majority of the time. For case b, an increase in oceanic yield strength results in generally fewer plates than in case a. Flow reversals associated with brief periods of zero surface mobility are observed at approximately 3.5 , 4.5 and 5.7 Gyr (the third reversal was observed in Fig. 2). CAID provinces are situated both distant from and beneath continents throughout the calculation period. For case c, an increase in continent yield strength results in similar behavior to case b for approximately $2 \mathrm{Gyr}$. Beyond this time, minor differences in continent count, plate count, surface mobility and province 
positions begin to occur. However, continent positions for cases $\mathrm{b}$ and $\mathrm{c}$ remain very similar until approximately 4.7 Gyr. At this time, a lower continent yield strength in case b allows for a supercontinent rupture while a higher continent yield strength in case $\mathrm{c}$ does not. For case c, large provinces exist both distant from and beneath continents until 6 Gyr. After that time, a single large province remains outside the subcontinental mantle until late continental aggregation (end of calculation). Motion in the same direction, of the single continent and CAID province, indicates that the province is incorporated within a distinct convection cell from the plate accommodating the continent.

Particularly in cases a and $\mathrm{b}$, there are long periods during which large provinces are essentially fixed in position, interspersed with periods of rapid province migration. In addition, the surface remains in the mobile-lid regime (although a few flow reversals are associated with very brief periods of zero surface mobility, the plate counts never reach unity). In general, there is a positive correlation between the plate count and surface mobility. Also, higher continent counts occur with a reduction in continent yield strength. Gradual mixing of compositional province material is observed which occurs due to viscous tractions present at the interface between compositionally enriched and ambient mantle material.

Figure 4 shows snapshots from five different calculations featuring different continental and oceanic yield stresses after $6.5 \mathrm{Gyr}$ of evolution. Each snapshot depicts the temperature, surface age and plate boundaries. In calculations featuring compositional heterogeneity and/or continents, composition and/or continent locations are also shown. For Model ON80_CT80_CH (top panel) there are two continents and six plate boundaries. There is a large compositional province featuring hot upwellings at its edges associated with divergent margins at the surface. There are also large, hot upwellings distant from the province, associated with a spreading center that has separated the two continents. There are two convergent margins, both of which are situated near the edges of continents. For Model ON100_CT100_CH, featuring increased oceanic and continental yield strengths, there is a supercontinent, two plate boundaries and a large compositional province. The convergent margin is positioned near the right edge of the supercontinent, while the divergent margin lies above a large upwelling situated to the right of the province. Surface age is generally higher compared to Model ON80_CT80_CH. Increased oceanic and continental yield strengths have resulted in the formation of fewer divergent margins and less new surface material. For Model ON100_CT80, there are 13 plate boundaries and two continents. We find the absence of compositionally heterogeneous provinces has allowed the organization of large, hot upwellings that frequently rupture the surface. Accord- 
ingly, oceanic surface age is young. For Model ON100_CTNA_CH, there are eight plate boundaries and a broad compositional province with highly variable topography. Finally, in Model ON100_CT80_CH* we find two continents and a single large province. In contrast, Model ON100_CT80_CH, which differs only by the initial placement of the continents, featured two CAID provinces at this time. These two cases are similar in that they oscillate between featuring one or two large CAID provinces but differ substantially in their supercontinent formation histories. Generally, the presence of dense compositional provinces results in weakening upwelling vigor as flow patterns become more time-dependent and active upwellings that are able to draw thermals from all parts of the adjacent thermal boundary layer are more difficult to form. The presence of continents appears to regulate province dimensions and morphology possibly because a smaller number of plate boundaries sweep the dense material into confined domains that are conducive to forming thick provinces. In all cases featuring compositional heterogeneity, the hottest upwellings are either distant from or at the edges of compositional provinces. In cases featuring continents, convergent plate boundaries tend to be near the edges of continents.

The panels in the top row of Figure 5 show the positions of the CAID material on the base of Model ON100_CT80_CH as a function of time (as in Fig. 3b). Continental positions are also represented. In addition, this figure shows the positions and magnitudes (by color) of cold anomalies and hot anomalies at depths of $300 \mathrm{~km}$ (left column) and $2500 \mathrm{~km}$ (right column). The panels in the bottom row of Fig. 5 show temperature anomaly time series as a function of position in Model ON100_CT80. The two cases presented feature the same parameters and initial conditions and differ only by the inclusion of the CAID component in the first calculation. In particular, Model ON100_CT80_CH exhibits a period of adjustment to the introduction of the CAID component. Following adjustment to the initial conditions this case is characterized by the presence of particularly hot and sustained thermal anomalies at the edges of the compositional provinces. However, hot anomalies can appear over CAID province interiors. The upwellings present at $2500 \mathrm{~km}$ depth arrive in the mantle above in pulses. Also evident in Model ON100_CT80_CH is a correlation between the positions of cold temperatures and the margins of the model continents and numerous deep hot anomalies in regions absent of the CAID material. For example, at approximate position 11,600 km and time 6.7 Gyr an upwelling appears in the lower mantle that ruptures the overlying supercontinent. In the shallow mantle the presence of the upwelling is marked by numerous pulses of hot material in roughly the same location. 
It should be noted that the broadness of the hot anomalies in the regions associated with CAID material is a result of the $2500 \mathrm{~km}$ depth at which the time slices are sampled. Upwellings embedded in the CAID provinces have not narrowed at this depth while upwellings rooted in the ambient mantle have narrowed into conduits above the thermal boundary layer. Thus, anomaly width in Fig. 5 is not an indication of upwelling vigor. Indeed, the array of upwellings that can be detected above the CAID province interiors at a depth of $2500 \mathrm{~km}$ at times $4 \mathrm{Gyr}$ and 7.5 Gyr result in the arrival of very few hot anomalies in the upper mantle (i.e., the interiors of the black regions contain relatively few (yellow) anomalies). Generally, hot anomalies in the upper mantle above CAID provinces in this model cluster close to the edges of these regions but interior hotspots can also occur.

The panels in the bottom row of Fig. 5 contrast with the calculation in which CAID provinces are present. In this case, upwellings can remain nearly fixed in position for periods exceeding billions of years of model time. (A few strong upwellings draw other developing thermals to a primary conduit.) In the absence of the CAID material time-dependence appears more muted. The presence of less transient upwelling positions appears to act as a barrier to the drift of overriding continents and reduces the total number of supercontinent formations. Indeed, continents that drift over robust upwellings in this calculation can be seen to rift into small fragments that then rapidly migrate across the surface. Supercontinents are therefore more difficult to form because larger continents cannot easily drift across the upwellings in these stable convection patterns. As in the previous case, downwellings tend to correlate with continental margins.

Figure 6a shows the mean age of the oceanic plates versus time for Models ON80_CT80_CH, ON100_CT80_CH, ON100_CT100_CH, ON100_CTNA_CH and ON100_CT80. All models are characterized by periods featuring the intermittent development of extremely old oceanic lithosphere (over $600 \mathrm{Myr}$ ) except for the case in which continents are absent (magenta curve). An increase in both oceanic and continental yield stress produces a clear trend towards greater amplitude and longer periods characterized by old surface age. In the pair of cases that differ only by the inclusion of a CAID mantle component (green and black curves) the presence of dense provinces has resulted in an increase in oceanic age. However, this set of calculations indicates that surface age is most profoundly amplified by the specification of higher yield stresses and the presence of continents. In Figure 6b, as expected, the model with the youngest mean surface age also has the coolest interior (magenta curve, case with no continents). Consequently, upwellings are more vigorous in this calculation due to stronger thermal contrast with the am- 
bient mantle. Temperatures increase as yield stresses are increased in cases featuring continents.

Figure 7a shows the percentage of basal coverage by CAID provinces versus time for Models ON80_CT80_CH, ON100_CT80_CH, ON100_CT100_CH and ON100_CTNA_CH. In all of these calculations, a general decrease in basal coverage over time is observed due to both the change in morphology of the initially uniform thickness compositional layer and the mixing of the two initially distinct mantle components resulting from the long-term process of viscous entrainment. Upwellings atop and at the edges of compositional provinces (e.g., Figs. 4 and 5) are sites of significant viscous entrainment resulting in erosion of the provinces over the billions of years that we model. Of the cases featuring continents, Model ON80_CT80_CH (red curve) has the least basal coverage at the end of the calculation period. In that model, an increase in plate count (e.g., Fig. 3) brought on by reduced oceanic and continental yield strengths has resulted in a cooler mantle (Fig. 6b). Consequently, upwellings are generally more buoyant, including the vigorous upwellings that form at the edges of large compositional provinces (e.g., Fig. 4) where substantial viscous entrainment occurs. For a given oceanic yield stress, calculations featuring continents have fewer plate boundaries and are hotter (provided that the continental yield stress is comparable to the oceanic). Consequently, upwellings in models with continents are relatively less buoyant and CAID provinces are preserved. For Model ON100_CTNA_CH (magenta curve), the absence of continents has lead to an increase in the rate of CAID province erosion resulting in $0 \%$ basal coverage by compositional provinces at 9.3 Gyr. Buoyant, vigorous upwellings have entrained more material and mixed the mantle components more effectively.

In Figure $7 \mathrm{~b}$ we plot time series of the integral (over the solution domain) of the compositionally dense component as a function of time. The time series correspond to the cases shown in Fig. 7a and indicate how mass loss due to accumulated numerical error impacts conservation of the CAID material. One possible source of error arises from the intermittent appearance of tracer free cells in the numerical grid. In these calculations, when cells become tracer-free, their composition is fixed to the value obtained when the cell last contained one or more tracers. We find that over the entire $10 \mathrm{Gyr}$ period that we simulate (in excess of 100 mantle transits), our calculations suffer approximately $2.5 \%$ loss of the original $5 \%$ of the volume filled by the CAID layer. For example, in Figure 7a, when 100\% of the CAID layer vanishes from the base of the system, $97.5 \%$ of the loss is accounted for by mixing due to phys- 
ical entrainment but approximately $2.5 \%$ of the loss may result from the accumulation of numerical error. In addition, we have performed a resolution test (see Appendix A).

Figure 8 shows time series similar to those in Fig. 3 but for Models ON100_CT80 (panel a), ON100_CTNA_CH (panel b) and ON100_CT80_CH* (panel c). Panel c features the same parameters and setup as in Fig. 3b except with different initial positions of the continents. Panel a illustrates model behavior in the absence of CAID material. Comparison of Fig. 8a and Fig. 3b shows that the presence of provinces changes the migration patterns of continents. In particular the frequency of the supercontinent formation is affected, as illustrated in the time series and discussed below. In panel $\mathrm{b}$, increased surface velocity leads to an increase in mixing and relatively rapid entrainment. For the buoyancy ratio used in this study, comparing Fig. 8b and Fig. 3b, it is observed that the presence of continents generally leads to fewer plate boundaries and lower surface mobility. This seems counterintuitive since for in Fig. 3b 30\% of the surface has a reduced tensile yield strength compared to $8 \mathrm{~b}$. However, each time a continental collision occurs a plate boundary is lost, thus reducing plate number. In addition, the inherent buoyancy of continents creates a tendency for convergent boundaries to locate at continental margins (e.g., Figs. 4 and 5). When both margins are the site of subduction, as is common when model supercontinents form, continents tend to remain fixed in position or migrate very slowly. The net influence of continents is that they reduce the entrainment rate of the provinces. In Fig. 8c prolonged periods of supercontinent assembly exist with a pair of extremely stable subcontinental and suboceanic CAID provinces present over the final 1.5 Gyr. A notable outcome of both Model ON100_CT80_CH and ON100_CT80_CH* is that they result in a pair of distinct CAID provinces for much of the latter part of their evolutions and that motion of the provinces is dramatically reduced during periods featuring supercontinent formation, which itself is associated with reduced continental motion in these models.

Figure 9 shows time series from Models ON100_CT100_CH (top row) and ON100_CTNA_CH (bottom row) presented in the same format as the panels in Fig. 5. The case in the top row is the same as the case in the top row of Fig. 5 except that the continental yield stress has been increased by $25 \%$. Accordingly, a supercontinent forms in Model ON100_CT100_CH that remains assembled for nearly 4 Gyr. During that period the continent drifts slowly over mantle in which CAID material is absent. At approximately $8.7 \mathrm{Gyr}$, subduction initiates on the left, as well as continuing on the right margin of the continent, and roughly $200 \mathrm{Myr}$ later the supercontinent finally rifts. Near the end of the calculation a new supercontinent assembles directly above the CAID province. During the previous period of supercontinent assembly, the 
single CAID province migrated across the base of the system at the same mean velocity as the continent. The cold thermal anomaly in Fig. 9 (top row) shows that the leading edge of the drifting continent over-rode a downwelling that is trailing the migrating dense province. Arrival of the downwelling at the base of the system thus appears to have pushed the province (left-to-right in the figure) ahead of the continent (which is drawn towards the convergent boundary by drag associated with the downwelling flow). Accordingly, the motion of the continent causes motion of the CAID province. While the province is in motion, upwellings forming in its interior (at $2500 \mathrm{~km}$ depth) appear to be as common as upwellings forming at the margins. However, hot anomalies arriving in the upper mantle don't appear to be related to interior upwellings. For example, when the motion of the province stalls at $9 \mathrm{Gyr}$, upper mantle anomalies above the province interior diminish in intensity while hotspots continue to appear close to the margins. Several strong upwellings form away from the CAID province but become swept up at its advancing margin. Between 6 and 9 Gyr, upwellings at the leading edge of the province produce interior province hot anomalies in the upper mantle as the entire province moves left to right in the figure. (Hot anomalies in the upper mantle start on the right-hand margin of the moving province and migrate across the interior as time increases.) Upwellings in the ambient mantle do not affect dense province motion. The long-lived supercontinent in the calculation eventually rifts over a robust upwelling unrelated to the dense province.

The bottom panels in Fig. 9 show the evolution of a model in which continents are absent. As noted previously, the absence of continents allows for particularly vigorous flow and the CAID layer becomes completely entrained. Once the CAID material is entrained a particularly fixed convection pattern is established. Prior to entrainment the CAID material typically disperses into one or two provinces and vigorous upwellings appear both at the margins and in the interiors of the provinces. However, hot anomalies appear in the upper mantle most frequently above CAID province margins.

Figure 10 shows time series of surface mobility for Models ON100_CT80_CH (red curve), ON125_CT80_CH (green curve) and ON125_CT80 (blue curve). These three models are representative cases from the three distinct mobility regimes found in this study (e.g., Table 1): mobile, intermittent and stagnant. Model ON100_CT80_CH remains in the mobile-lid regime. There are brief periods of zero mobility due to the limitations of a 2D model and changes in convection pattern forcing flow reversals, but the plate count remains above unity throughout the calculation (e.g., Fig. 3b). Model ON125_CT80_CH features a long period when the plate count is one, from approximately 8.5 to 9.7 Gyr. Subsequently, the surface ruptures resulting 
in nonzero mobility. We categorize such cases as being examples of an intermittent mobility regime. Model ON125_CT80 exhibits zero surface mobility after dropping to a plate count of one at 3.7 Gyr. In this case, surface stresses remain too low to rupture the surface and a stagnant lid is obtained [e.g., Moresi and Solomatov, 1998]. As the yield stress is increased both the intermittent and stagnant-lid regimes are encountered [e.g. van Heck and Tackley, 2011; Weller and Lenardic, 2012].

Figure 11a plots the time-averaged number of large provinces in oceanic/continental yield strength parameter space for all calculations featuring compositional heterogeneity (e.g., Table 1). At all times contributing to the average, for each CAID province, from largest to smallest, the contribution to the basal coverage is tallied until that sum is greater than or equal to $80 \%$ of the total basal coverage of all provinces. The number of provinces needed to meet this criterion is considered to be the number of large provinces at that time. As in Figs. 3, 5, 8 and 9, compositional provinces are required to have a minimum width and composition of $0.1 d$ (290 km) and $0.3 \Delta \rho_{C}\left(22.5 \mathrm{~kg} \cdot \mathrm{m}^{-3}\right)$, respectively. Based on findings in the calculations featuring continents with an oceanic yield strength of $100 \mathrm{MPa}$, we conclude that continent yield strength has little effect on the average number of large provinces. For calculations with oceanic yield strengths of both $100 \mathrm{MPa}$ and $125 \mathrm{MPa}$, the presence of continents increases the average number of large provinces. However, this trend is not observed for the calculations featuring $80 \mathrm{MPa}$ oceanic yield strength. In general, all calculations featuring compositional heterogeneity are structurally dominated by 1 to 2 large provinces.

Figure 11b shows the number of supercontinent assemblies in continental/oceanic yield strength parameter space for all pairs of calculations (with and without CAID material) featuring continents (e.g., Table 1). A supercontinent assembly occurs when all continental material coalesces through collisions into a single continent. Supercontinent ruptures do not always result in long-lived multicontinent structures (e.g., Fig. 3c at 2.5 and $7.5 \mathrm{Gyr}$ ). To avoid considering such instances as supercontinent breakup events we require that multicontinent eras exceed a minimum of $0.1 \mathrm{Gyr}$. Consistently, calculations featuring compositional provinces result in substantially increased assembly counts compared to isocompositional calculations. In fact, for equivalent continental and oceanic yield strengths, calculations with provinces frequently show twice the number of assemblies compared to those cases without compositional heterogeneity.

Figure 11c summarizes the temporally-averaged oceanic ages in each calculation in the mobile-lid regime. The presence of CAID provinces increases surface age in all pairs of mod- 
els that differ only by the inclusion of the CAID component. Thus oceanic age increases along with the frequency of supercontinent assembly. This finding is consistent with work previously presented by Trim et al. [2014] that found surface mobility and plate number are decreased by the presence of an intrinsically dense component in the mantle because the dense material diminishes upwelling vigor and the contribution of positive buoyancy to stresses in the lithosphere. However, a decrease in surface velocity does not preclude decreased stability of convection patterns and increased system time dependence.

\section{Discussion}

\subsection{Model considerations}

We have not considered variable density contrasts (i.e., $B=0.7$ is the only buoyancy ratio specified) between the CAID provinces and ambient mantle nor a unique rheology for the dense provinces. The choice of the buoyancy ratio adopted in our calculations is somewhat constrained by the model dynamics. Previously, Trim et al. [2014] showed that if $B \geq$ 0.8 the CAID component settles into a nearly uniform thickness layer on the CMB. For $B \leq$ 0.6 material mixing is relatively rapid and stable provinces are difficult to obtain on the timescales considered in this study [e.g., Le Bars and Davaille, 2004b]. The inclusion of compositiondependent rheology will likely affect how actively the CAID provinces deform during model evolution [e.g., McNamara and Zhong, 2004a]. However, the rheological properties of compositionally distinct provinces in the Earth (the possible origin of LLSVPs) are not currently constrained. The influence that a compositionally-dependent viscosity has on CAID provinces and the supercontinent cycle should be the subject of further investigation as possible rheological constraints are identified.

In this study the volume of the CAID material is fixed. (Differences in entrainment rates are a result of the independent parameters specified (i.e., yield stress values and the presence of continents).) Thus, when compositional heterogeneity is present, differences in model behavior are more apparent in the latter stages of calculation evolution. This is partially due to the different rates of entrainment of CAID material. A different initial distribution of CAID material may also affect entrainment rates and system behavior.

Although we have not modeled a fully temperature-dependent viscosity, our methodology captures the first order effect of the increase and decrease in viscosity at the upper and lower thermal boundary layers, respectively. The influence of thermal boundary layer viscosity on boundary layer stability is therefore modeled so that, with our approximation, upwellings 
may be expected to form similarly to how they would for a fully temperature-dependent rheology. Nevertheless, our omission of the effect of modeling low viscosity plume conduits and heads will likely influence upwelling rise times and the contributions of active upwellings to stresses on the plates. The latter effect should influence continental breakup and the rupture of oceanic plates. However, for similar ratios of continental to oceanic strength, we expect similar trends to prevail to those described here. Modeling a temperature-dependent viscosity is expected to influence a baseline yield stress magnitude required for regularly occurring continental breakup. Thus the 80-100 MPa range of yield stresses that allows for supercontinent cycles and surface mobility in this study may differ from a range providing similar behavior in a model with a fully temperature-dependent rheology.

The implementation of temperature-dependent variables such as viscosity requires the modeling of an appropriate geotherm. In order to obtain geotherms similar to those obtained in a bimodally heated spherical shell geometry (see Fig. 1), we have used a non-dimensional heating rate of zero [O'Farrell and Lowman, 2010; O'Farrell et al., 2013]. The thermal contrast between the plumes and CAID provinces modeled in this work will therefore be similar to the temperature contrasts found in a spherical geometry with internal heating rates similar to the present-day Earth. However, we have not modeled the secular cooling that occurs due to the long-term extraction of heat from the core nor the reduction, in time, of the concentration of radioactive isotopes in the mantle. Indeed, our model features numerous idealizations relative to the convecting terrestrial mantle, but our modeling approach does allow for isolation of the effect of incorporating a compositionally dense component in the lower mantle and determining its impact on surface dynamics.

Our finding that the presence of CAID material increases system time-dependence (specifically, increasing upwelling mobility, plate rupturing events and supercontinent assemblies) contrasts with some previous studies that found a dense bottom layer stabilizes plumes in mantlelike systems. For example, in thermochemical convection experiments featuring a similar Rayleigh number and buoyancy ratio to our model setup, Jellinek and Manga [2002] found that the presence of intrinsically dense material resulted in very steady plume positions relative to that found in isochemical convection. However, our contrary findings are for systems featuring a freeslip base and much larger aspect ratio (10 versus 2$)$ than the former study. In addition, the inclusion of plate-like surface motion, continents, and plate boundary migration resulting in onesided subduction, each act as sources of heterogeneity in the upper thermal boundary layer of our calculations. These surface heterogeneities interfere with the formation of a steady sur- 
face planform in general but do not explain why we find decreased time-dependence in an isochemical system. However, our observations agree with those of McNamara and Zhong [2004b] who found plumes are more fixed in isochemical cases in two-dimensional systems with freeslip bases. McNamara and Zhong [2004b] also found that plume steadiness in 3D isochemical and thermochemical convection cases is more similar. The limitation of our study to two dimensions may therefore also explain some of the contrast between our findings and those of Jellinek and Manga [2002]. Future studies combining the most desirable elements of the 2D and 3D models are clearly needed.

Nevertheless, it is currently computationally prohibitive to achieve the grid and tracer resolution necessary to include Earth-like mantle parameters in global scale three-dimensional thermochemical calculations featuring continents and plates. In particular, the necessary integration times to study supercontinent cycles in 3D high Rayleigh number calculations with a compositionally heterogenous mantle are impractical. However, the use of a two-dimensional geometry can lead to behavior that may not be observed in a three-dimensional spherical shell. (For example, plate direction reversals characterized by surface mobility that briefly drops to zero followed by a $180^{\circ}$ change in plate velocity direction. This behavior is unlikely to occur in three dimensions [Lowman et al., 2003].)

\subsection{Application to Earth's evolution}

In all calculations featuring compositional heterogeneity, dense material generally forms one or two large provinces (e.g., Fig. 11a). We find the arithmetic average of the temporallyaveraged number of large CAID provinces is 1.47 . The arithmetic averages with and without continents are 1.56 and 1.39 , respectively. For the majority of cases, we find that the number of large provinces increases in the presence of continents. Given that neither the number nor the length scale of the CAID provinces in the calculations is specified, this finding determined by dynamics shows that the aggregation of an intrinsically dense mantle component into length scales consistent with the presently observed LLSVPs is compatible with estimates of the Rayleigh number and length scale of the Earth's mantle.

One feature appearing in some of our calculations, that may be exaggerated relative to terrestrial evolution, is the intermittent development of extremely old oceanic lithosphere (e.g., sections of old ocean appear in Fig. 2, at 6.0 and 6.2 Gyr and Fig. 4 Model ON100_CT100_CH). The observed ages are, in some cases, due to plate velocity reversals If a plate reversal takes place, oceanic lithosphere that would have been subducted remains at the surface only to re- 
traverse the region between the adjacent plate boundaries (while continually increasing in age). Previous studies have concluded that plate reversals in 2D models are an example of rapid plate velocity direction changes observed in some 3D models [Monnereau and Quéré, 2001; King et al., 2002; Lowman et al., 2003]. In studies of changes in plate velocity in three-dimensions, decreases in plate velocity frequently precede the directional change. However, in two dimensions the change in velocity magnitude is exaggerated because only a $180^{\circ}$ change can occur. Consequently, surface ages become amplified on reversing plates. Older oceanic plates can also develop when yield stresses are increased so that the number of plate boundaries and the frequency of continental breakup decreases. More frequent continental breakup results in younger oceans (e.g., compare Models ON80_CT80_CH and ON100_CT100_CH in Figs. 3 and 4). In addition to the dynamic affects on the ages obtained in our calculations the absolute magnitudes of the times quoted are potentially specious due to some uncertainty in the globally representative value of thermal diffusivity that we adopt (i.e., our assumption that $\kappa=10^{-6} \mathrm{~m}^{2} \mathrm{~s}^{-1}$ assigns a widely quoted order of magnitude value for the mantle to the single thermal diffusivity value used for our model but not a precise value). Both velocities and times presented will be in error by the same percentage as the error on the adopted diffusivity value. However, the ages and velocities are indicative of the order of magnitude and relative time scales involved in the processes modeled.

Despite the fact we ignore the effect of secular cooling, given the thermal diffusivity value adopted in our calculations, we model periods comparable to and even exceeding the age of the Earth. We integrate over many mantle overturns in order to sample the temporally prevalent dynamics rather than possibly atypical episodes but we do not attempt to model the Earth's thermal evolution. Instead, our goal has been to observe the interaction between surface evolution and CAID provinces and to identify possible trends and correlations. For completeness we have therefore considered contrasting cases in which either continents or CAID provinces are absent. Each of our calculations starts with common surface ages and plate boundary locations and, in the cases featuring an intrinsically dense component, emplacement of a highly idealized distribution of the compositionally anomalous layer. Accordingly, for most of the models, the evolution of the first $10-15 \%$ of the times modeled are qualitatively similar. Moreover, much of the first quarter of the evolution acts as an adjustment period in each calculation, during which the initial CAID layer organizes into one of more provinces. Typically basal coverage of the system is approximately $70 \%$ at this time (Fig. 7) and the evolution of the different cases presented becomes more distinct subsequently. 
The results presented in Fig. 7 show that the presence of continents not only has an impact on the basal coverage of CAID provinces but also the loss of the provinces. Short-term fluctuations in basal coverage occur due to changes in province morphology, arising due to the impact of upwelling and downwelling dynamics. However, a gradual long-term decrease in basal coverage occurs due to the viscous entrainment of material on the interface between the CAID provinces and ambient mantle. Entrainment arises from the tractions on the compositionally dense material by the stresses associated with adjacent buoyant mantle [Davaille, 1999]. We find that the presence of continents decreases the rate of entrainment, leading to increased longevity of the compositional anomalies.

Supercontinent formation disrupted by periods of dispersal was found in all of our calculations featuring yield stresses of $100 \mathrm{MPa}$ and less. In comparisons of pairs of models that differ only by the inclusion of a CAID mantle component versus a compositionally homogeneous mantle, we found that the CAID component increased model time-dependence (e.g., Figs. 3b and 8a) and the frequency of supercontinent formation (e.g., Fig. 11b). Overall, we find that on time scales of hundreds of millions of years, a CAID component increases system timedependence rather than stabilizing convection patterns. (Specifically, our systems featuring a CAID component fluctuate between periods characterized by low surface mobility and rapid surface motion (note the time series in Figs. 3b and 8a).) A possible explanation for the increased time-dependence is the feedback between two compositional heterogeneities in the boundary layers of the calculations. When continents migrate they can force migration of convergent plate boundaries and therefore downwellings. We find downwellings can drive the motion of CAID provinces which influence the mobility and formation of upwellings. Increased upwelling mobility prevents the formation of fixed convection patterns that can allow for prolonged periods of continental dispersal (e.g., see the long term fixity of the upwellings in the bottom right of Fig. 5).

Although CAID provinces are capable of migration at velocities comparable to continental drift, lengthy periods of province fixity are often observed (e.g., in Fig. 5a, a single fixed province exists between 7 and 8 Gyr and two nearly stationary provinces are present between 8.5 and $9.5 \mathrm{Gyr}$ ). Moreover, these periods of province immobility are characterized by the presence of sustained upwelling sites at the margins of the provinces. Our findings are consistent with studies that have mapped the mobility of inferred plume generation zones associated with the edges of LLSVPs. Using LIP eruption sites to infer plume locations, Torsvik et al. [2010] conclude that the edges of present-day compositionally distinct provinces have been fixed for 
200-540 Myr. Our calculations feature periods of province fixity on such time scales, however, we did not find examples of province fixity on time scales comparable to the age of the Earth. Frequently, we found fixed province positions would appear under oceanic regions, however, we also saw examples of supercontinent assembly over a CAID province that subsequently fragmented prior to eventual continental breakup (see Model ON100_CT80_CH between 8 and 9 Gyr). Generally, we observed that periods of province fixity exist on a similar time scale to periods of supercontinent immobility (Fig. 3). When continental motion is present (and typically driving migration of a convergent plate boundary) CAID provinces also respond by moving, though often after some lag.

CAID material location versus continental position is summarized in Figure 12. Figure 12a provides time series of statistics illustrating CAID province positions versus continental lithosphere locations for Model ON100_CT80_CH. The green curve plots the suboceanic percentage of basal CAID material. The red curve shows the percentage of the subcontinental base of the system that is covered by CAID material. The time series show distinct behavior beyond a transition that occurs at approximately 4 Gyr. After this time distinct well-formed CAID provinces have evolved e.g., 3 and the green curve shows that one or two provinces are often found only below oceans (e.g., from 6.6 to $7.8 \mathrm{Myr}$ ). However, the red curve shows that periods do occur where large fractions of the subcontinental lithosphere overlies a CAID province (e.g., between 7.8 and 9.4 Myr). Figure 12b summarizes the temporal averages of the distribution of CAID provinces below the continents and oceans in all calculations featuring both continents and CAID material. For each oceanic yield stress one Model is represented by a square and diamond (i.e., results from six models are plotted). The squares indicate the mean percentage of the subcontinental area covered by CAID material (e.g., average of a red curve in Fig. 12a). Diamonds show the percentage of the total area covered by CAID material that lies below oceanic lithosphere (average of a green curve like in Fig. 12a). As a time-average, we find more than $80 \%$ of the area covered by CAID material lies below the oceans in all cases. Thus the majority of CAID coverage of the base of the system is suboceanic. Accordingly, we find less than $50 \%$ of the subcontinental mantle base is covered by anomalously dense material. In general, we therefore conclude that the locations of CAID provinces and continental lithosphere do not appear to be positively correlated. However, CAID material is often present below continents. As indicated by Fig. 12a, Figs. 3 and 8c indicate that CAID material is found below continents less often in the latter stages of our calculations, well after the formation of distinct provinces has occurred. Thus, some late stage anti-correlation may be common. How- 
ever, in the latter stages of Model ON100_CT80_CH both suboceanic and subcontinental provinces are present (Fig. 8c).

Accordingly we conclude that our calculations do not show a correlation between the positions of CAID provinces and supercontinents. However, supercontinent assembly frequency and continental migration are influenced by the presence of CAID provinces. Our results show that when CAID provinces are present the number of supercontinent assemblies is increased in comparison to calculations with an isocompositional mantle. In general, system time-dependence is increased when the lower mantle's composition is heterogeneous (e.g., note the variability in surface mobility in Fig. 3b versus Fig. 8a).

Large igneous provinces and kimberlites have been identified with the eruption of mantle plumes originating at the CMB and appear to be correlated with the locations of the LLSVPs [Austermann et al., 2014]. We observe that the margins [Burke et al., 2008] of the CAID provinces in our calculations are consistently sites of active upwelling formation (e.g., Figs. 5 and 9) and produce associated upper mantle temperature anomalies. We also observe the formation of intraprovince upwellings (e.g., Fig. 5a, right panel, times 4 Gyr and $7.5 \mathrm{Gyr}$ ) but find these upwellings are rarely vigorous enough to reach the upper mantle (e.g., Fig. 5a, left panel, times 4 Gyr and $7.5 \mathrm{Gyr})$.

In models featuring CAID material, individual continents are observed to have higher drift rates following a supercontinent breakup. An intrinsic feature of the supercontinent cycle is variability in the actual record of motion of cratons. While it was a component of Gondwana, India's drift rate relative to the fixed hotspot reference frame was on the order of 6 to $8 \mathrm{~cm} / \mathrm{yr}$ [Scotese and McKerrow, 1991], similar to the other cratons comprising Pangea's southern component. However, since the breakup of Gondwana, India has virtually raced across the Earth's surface at velocities reaching 18 to 20 cm/yr [Kumar et al., 2007]. Our Model ON100_CT80_CH

(Fig. 2 and Fig. 3b) shows substantial increases in surface velocity occur following supercontinent breakup at $t=4.7,6.1$ and $10 \mathrm{Gyr}$ with continental velocities reaching $10 \mathrm{~cm} / \mathrm{yr}$ (given our adopted thermal diffusivity value). Comparable variability in mobility does not appear in an otherwise identical model in which compositional heterogeneity is absent (Model ON100_CT80, Fig. 8a).

\section{Conclusions}

Although a multitude of possibilities exist for the dispersement of a dense compositionally distinct mantle component lying on the $\mathrm{CMB}$, in agreement with the present-day distri- 
bution of the LLSVPs, in all of our calculations we find that CAID material frequently forms two continuous provinces and tends to alternate between forming one or two large complexes. Occasionally we observe the presence of additional finer structures, a wide distribution of smaller piles or wide provinces with highly variable topography (e.g., Fig. 2 at $5.0 \mathrm{Gyr}$ ). However, 1-2 large provinces is the most common distribution.

We did not find any combinations of oceanic or continental plate yield stresses that result in fixity of the compositionally dense provinces. However, we did find that nearly stationary CAID provinces can form on 1 Gyr time scales. For example, in Model ON100_CT80_CH* (Fig. 8c) fixity prevails for the final $2 \mathrm{Gyr}$ of the calculation. (In this case an almost stationary supercontinent formed throughout the same period.) In general, periodically immobile provinces are a common finding, however, CAID province mobility is similarly common. Regardless of motion, for the yield stress combinations considered, we find isolated (and often mobile) provinces can persist for periods exceeding a billion years (e.g., Model ON100_CT100_CH).

The supercontinent cycle is fundamentally influenced by the presence of CAID material and lithospheric strength determines the distribution of CAID province material. For example, when we increase oceanic lithospheric strength to $125 \mathrm{MPa}$, intermittent periods of surface stagnation occur. Periods of prolonged surface stagnation result in spreading of the CAID layer over the base of the system and dampening of the vigor of mantle upwellings [Trim et al., 2014] so that the CAID material's presence allows the formation of a positive feedback loop that promotes stagnation. In contrast, when the continental lithosphere yields, the resulting motion of downwellings contributes to sweeping CAID material into distinct provinces that play a role in upwelling formation. Specifically, the evolution of the morphology and position of the CAID provinces adds to system time-dependence and the margins of the provinces can form generation points for active thermal upwellings that penetrate the upper mantle. Accordingly, for the volume of anomalously dense material considered in this study, the presence of CAID material enhances system time-dependence (e.g., it diminishes periods over which flow patterns become established) and promotes more frequent supercontinent assembly and breakup (Fig. 11b). 
Table 1. Model Summary ${ }^{a}$

\begin{tabular}{lccc}
\hline Model Name & Mobility Regime & Average \# of Large Provinces & Supercontinent Assemblies \\
\hline ON80_CTNA & M & NA & NA \\
ON80_CTNA_CH & M & 1.466 & NA \\
ON100_CTNA & M & NA & NA \\
ON100_CTNA_CH & M & 1.356 & NA \\
ON125_CTNA & M & NA & NA \\
ON125_CTNA_CH & M & 1.359 & NA \\
ON100_CT50 & M & NA & 1 \\
ON100_CT80 & M & NA & 3 \\
ON100_CT100 & M & NA & 2 \\
ON125_CT80 & S & NA & 2 \\
ON80_CT80_CH & M & 1.368 & 5 \\
ON100_CT50_CH & I & 1.559 & 5 \\
ON100_CT80_CH & M & 1.534 & 7 \\
ON100_CT100_CH & M & 1.545 & 4 \\
ON125_CT80_CH & I & 1.591 & 6 \\
ON100_CT80_CH* & M & 1.646 & 3 \\
\hline
\end{tabular}

${ }^{a}$ Model names are described in the text. Surface mobility regimes are indicated by either M, I or $\mathrm{S}$ for mobile lid, intermittent or stagnant lid, respectively. The time-averaged number of large compositional provinces (described in the text) and supercontinent assembly count is also shown. Supercontinent assemblies interrupted by breakup periods that last less than $100 \mathrm{Myr}$ are considered periods of continuous assembly (see discussion of Fig. 11b). Entries that are not applicable based upon model features are indicated by NA. 
a) $\mathrm{A}$

A

T
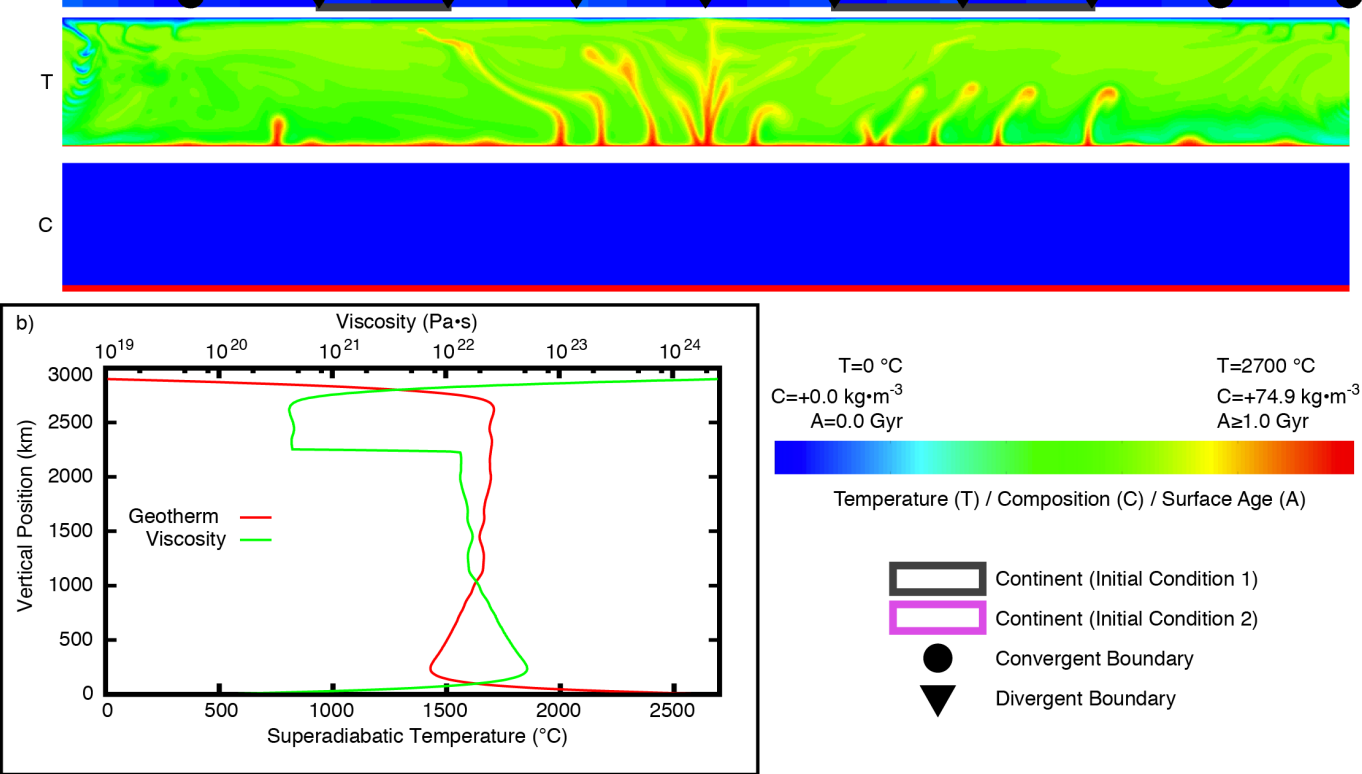

$\mathrm{T}=2700^{\circ} \mathrm{C}$

$\mathrm{C}=+74.9 \mathrm{~kg} \cdot \mathrm{m}^{-3}$ $\mathrm{A} \geq 1.0 \mathrm{Gyr}$

Figure 1. Initial surface age (A), superadiabatic temperature (T) and composition (C) fields at 0 Gyr. Initial positions of convergent and divergent plate boundaries are represented by black circles and triangles, respectively. In calculations featuring continents, initial continent positions are outlined in dark gray /bf in the lower surface age plot. In calculations denoted by a superscript asterisk, the initial continent position is outlined in magenta in the upper surface age plot. 

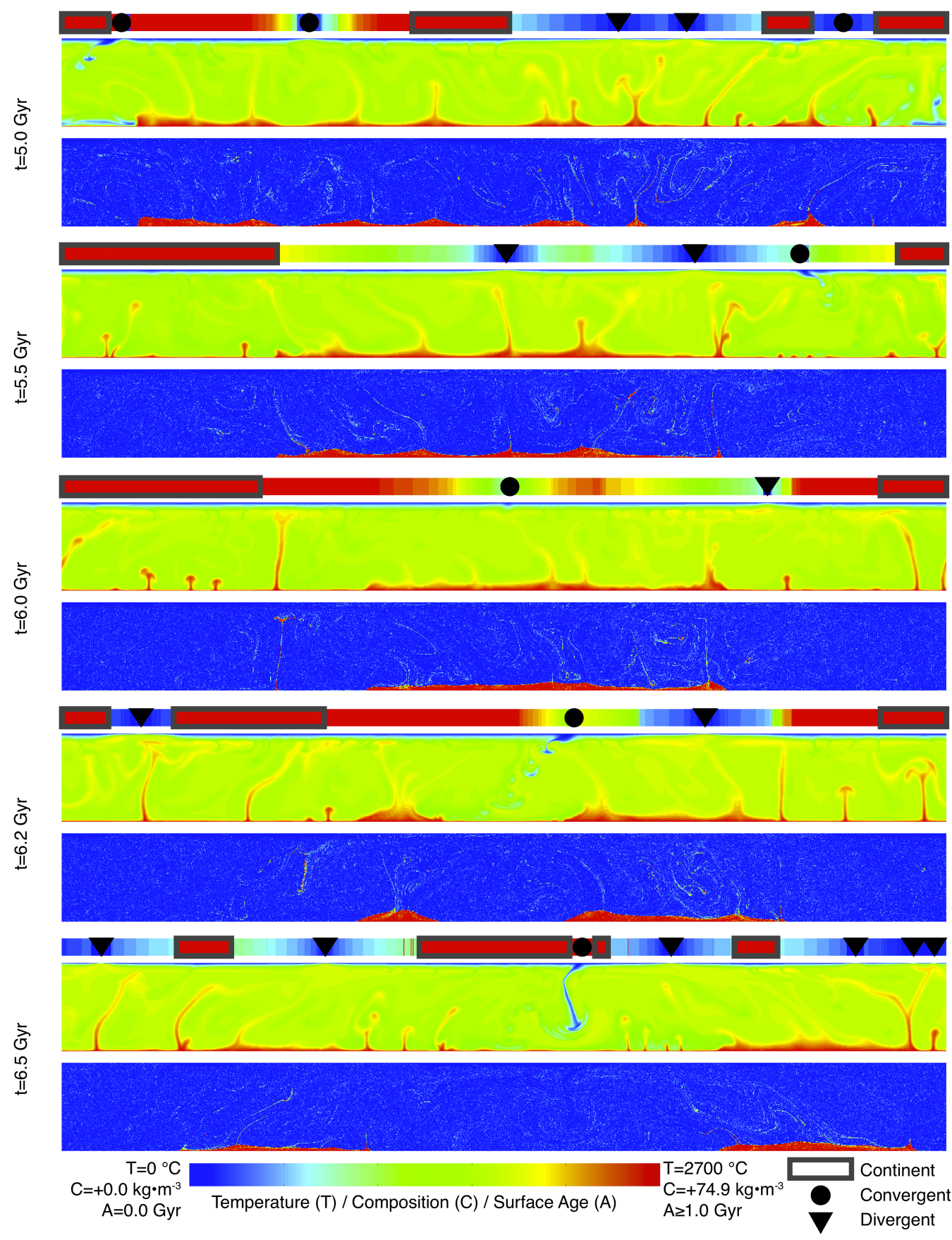

Figure 2. Snapshots of surface age, superadiabatic temperature field and composition at selected times for Model ON100_CT80_CH. The positions of convergent and divergent plate boundaries are represented by black circles and triangles, respectively. Continent positions are outlined on the surface age plot in dark gray. 


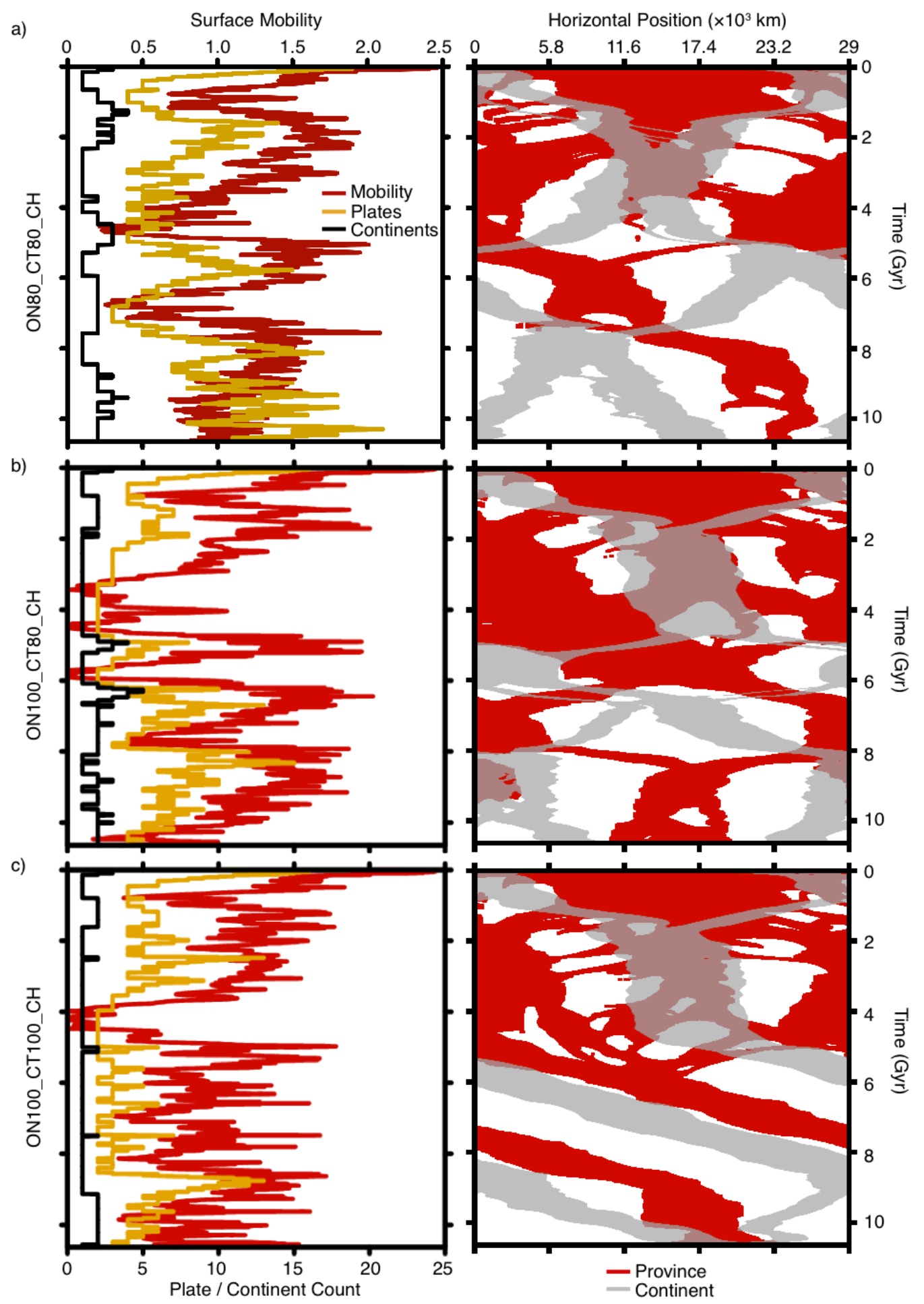

a)

b)

c)

Figure 3. Time series from Models a) ON80_CT80_CH, b) ON100_CT80_CH and c) ON100_CT100_CH.

For all plots, time is indicated on the vertical axes. Time series of surface mobility, plate count and continent count are shown in the left column of parts a, b and c. Time series of surface coverage by continents and system basal coverage by compositional provinces are shown in the right columns. 

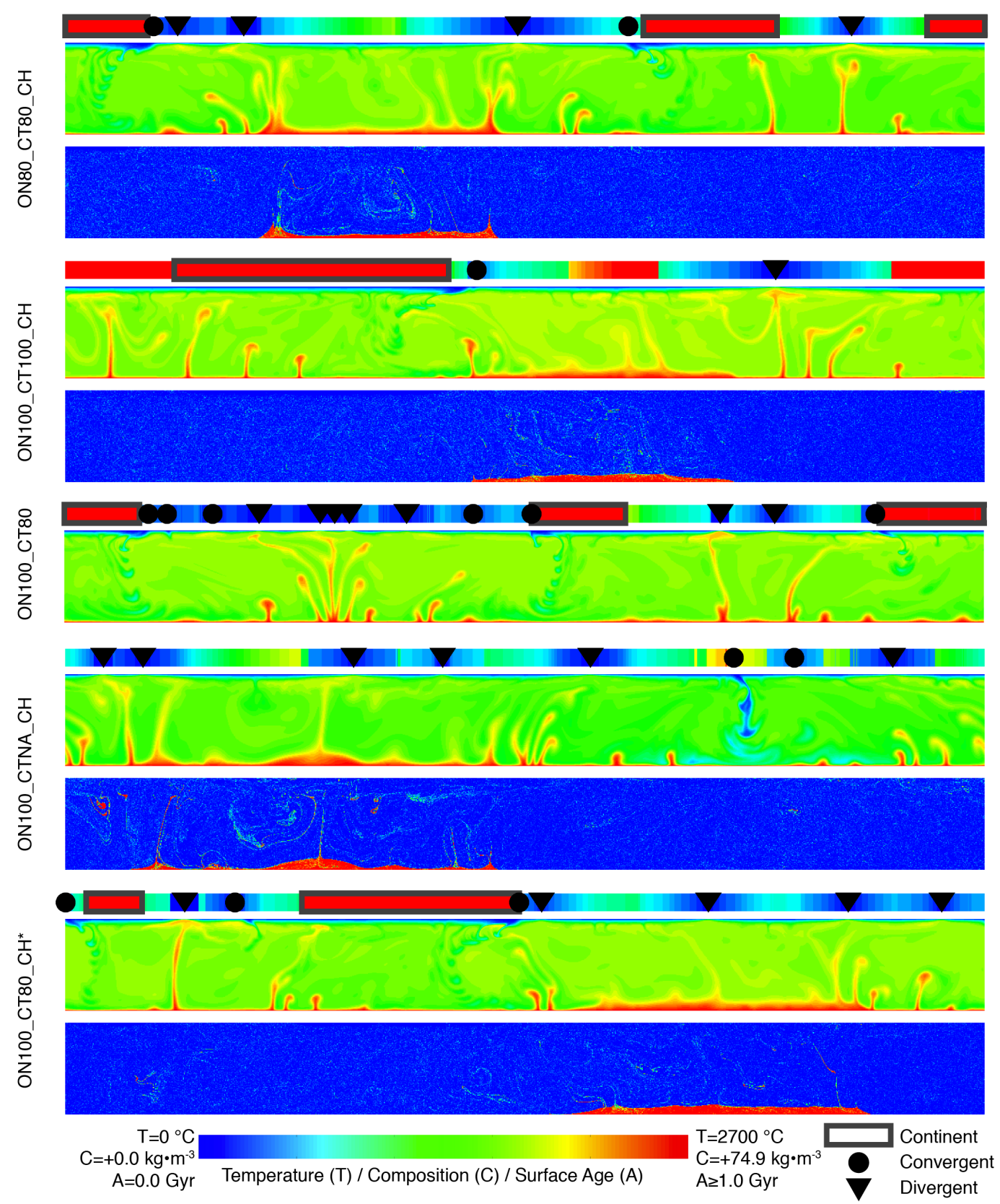

Figure 4. Surface age and superadiabatic temperature fields at 6.5 Gyr for Models ON80_CT80_CH, ON100_CT100_CH, ON100_CT80, ON100_CTNA_CH and ON100_CT80_CH*. The positions of convergent and divergent plate boundaries are represented by black circles and triangles, respectively. In Models featuring continents, continental positions are outlined on the surface age plots in dark gray. For Models with a $\mathrm{CH}$ suffix, corresponding composition fields are also shown. 

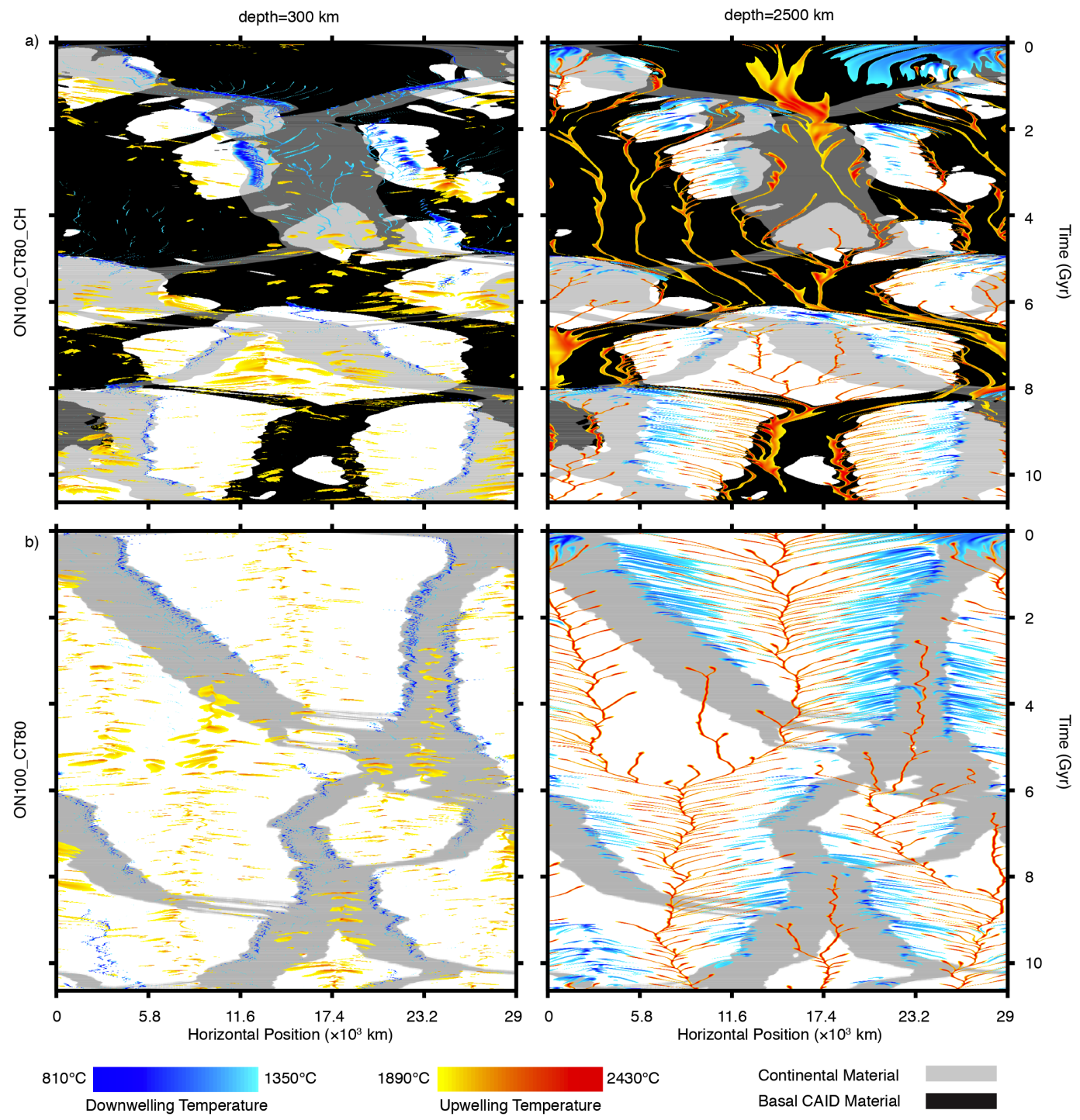

Continental Material

Figure 5. Time series of basal coverage by CAID material (black), continent positions (gray) and fixed depth positions of cold (downwelling) and hot (upwelling) superadiabatic temperature anomalies. Time increases on the vertical scales from top to bottom. Superadiabatic temperature anomalies are shown from left to right at depths of $300 \mathrm{~km}$ (left column) and $2500 \mathrm{~km}$ (right column) in Models ON100_CT80_CH (top row) and ON100_CT80 (bottom row). 

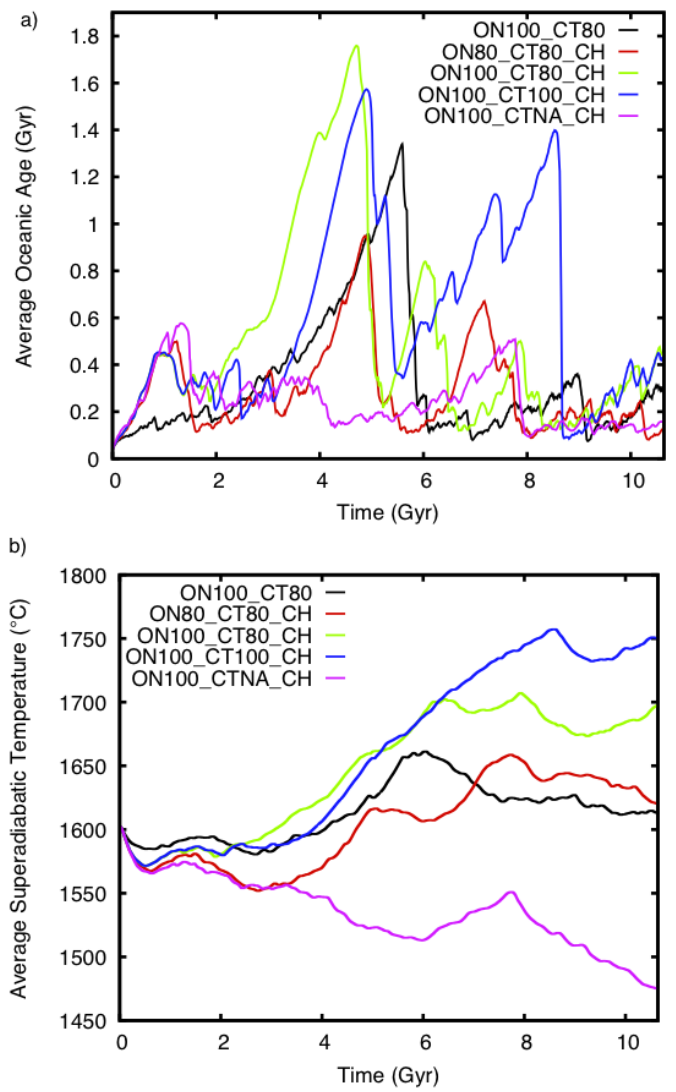

Figure 6. Time series of: a) mean oceanic plate age; and b) mean temperature; in Models ON80_CT80_CH, ON100_CT80_CH, ON100_CT100_CH, ON100_CTNA_CH and ON100_CT80. 

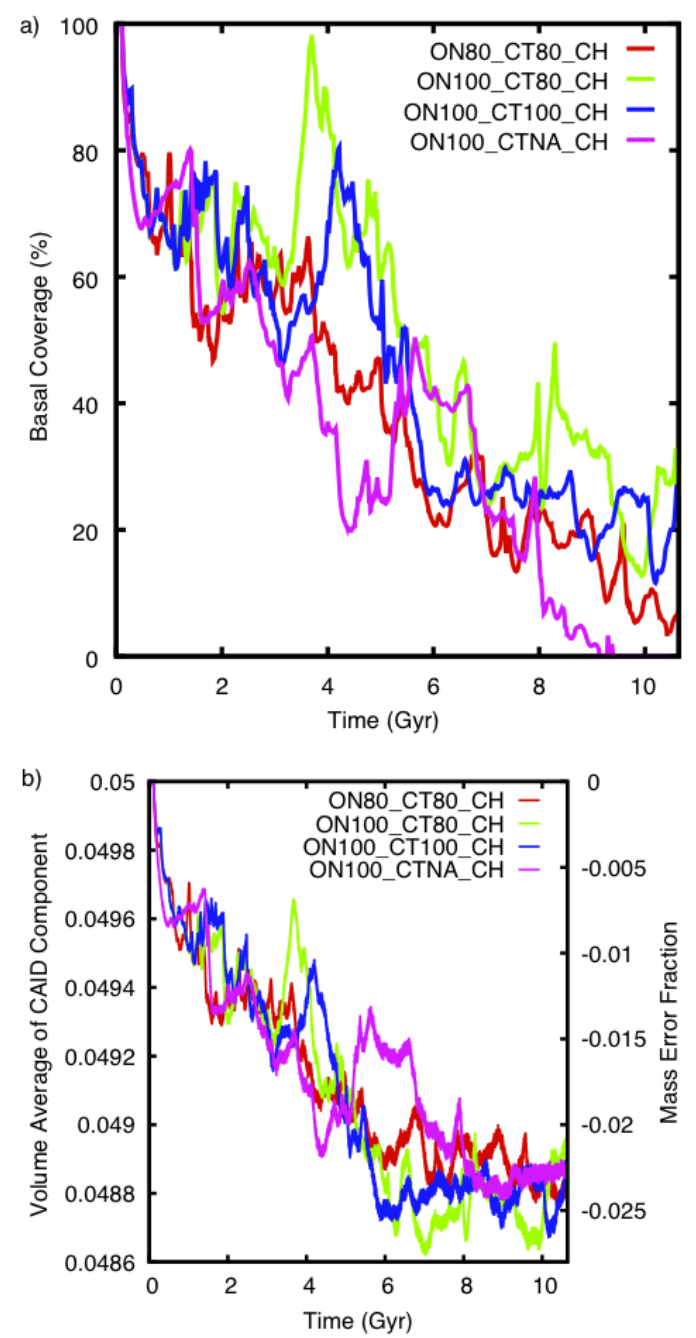

Figure 7. Time series for Models ON80_CT80_CH, ON100_CT80_CH, ON100_CT100_CH and ON100_CTNA_CH of a) basal coverage by compositional provinces and b) the volume average of CAID material. For part b), the mass error fraction is the difference in total CAID material from the initial amount, divided by the initial amount. 


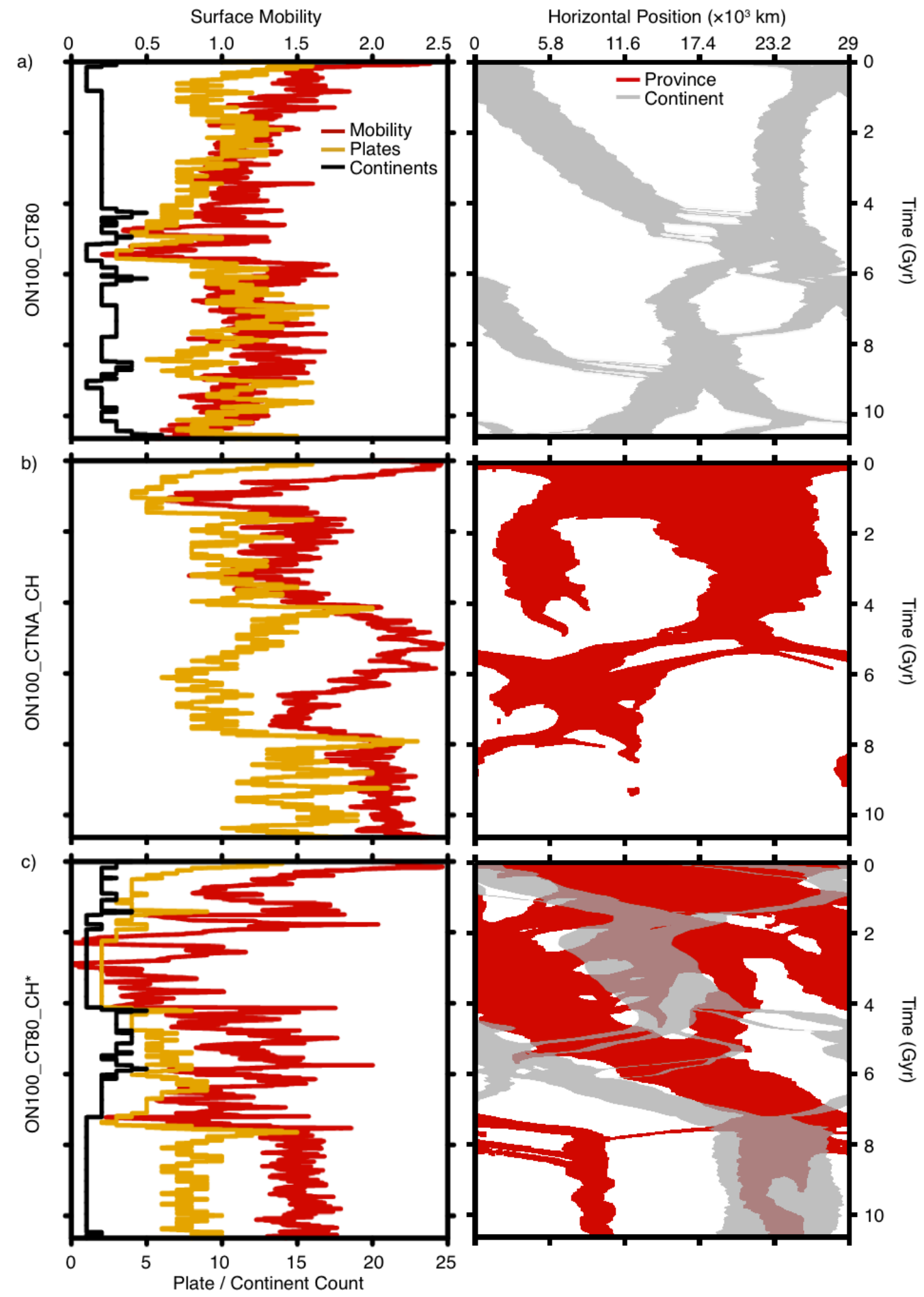

Figure 8. Time series as in Fig. 3 but for Models a) ON100_CT80 (featuring no compositional heterogeneity), b) ON100_CTNA_CH (featuring no continents) and c) ON100_CT80_CH* (featuring a different placement of the continents at time $t=0)$. 

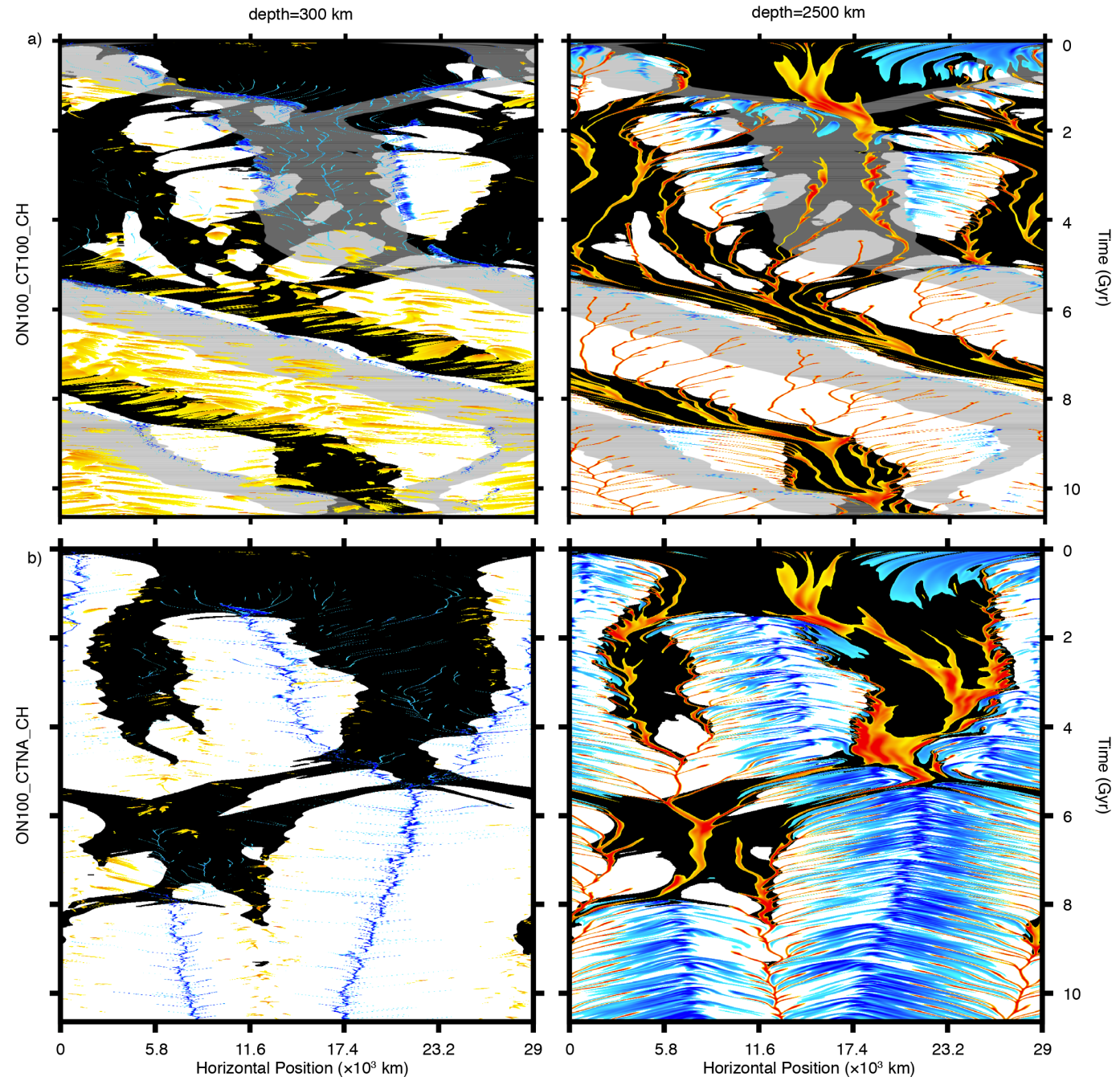

\begin{tabular}{c|c|c|c|}
$810^{\circ} \mathrm{C}$ & $1350^{\circ} \mathrm{C}$ & $1890^{\circ} \mathrm{C}$ & \\
Downwelling Temperature & $2430^{\circ} \mathrm{C}$
\end{tabular}

Continental Material Basal CAID Material

Figure 9. Time series as in Fig. 5 but for Models a) ON100_CT100_CH and b) ON100_CTNA_CH (featuring no continents). 


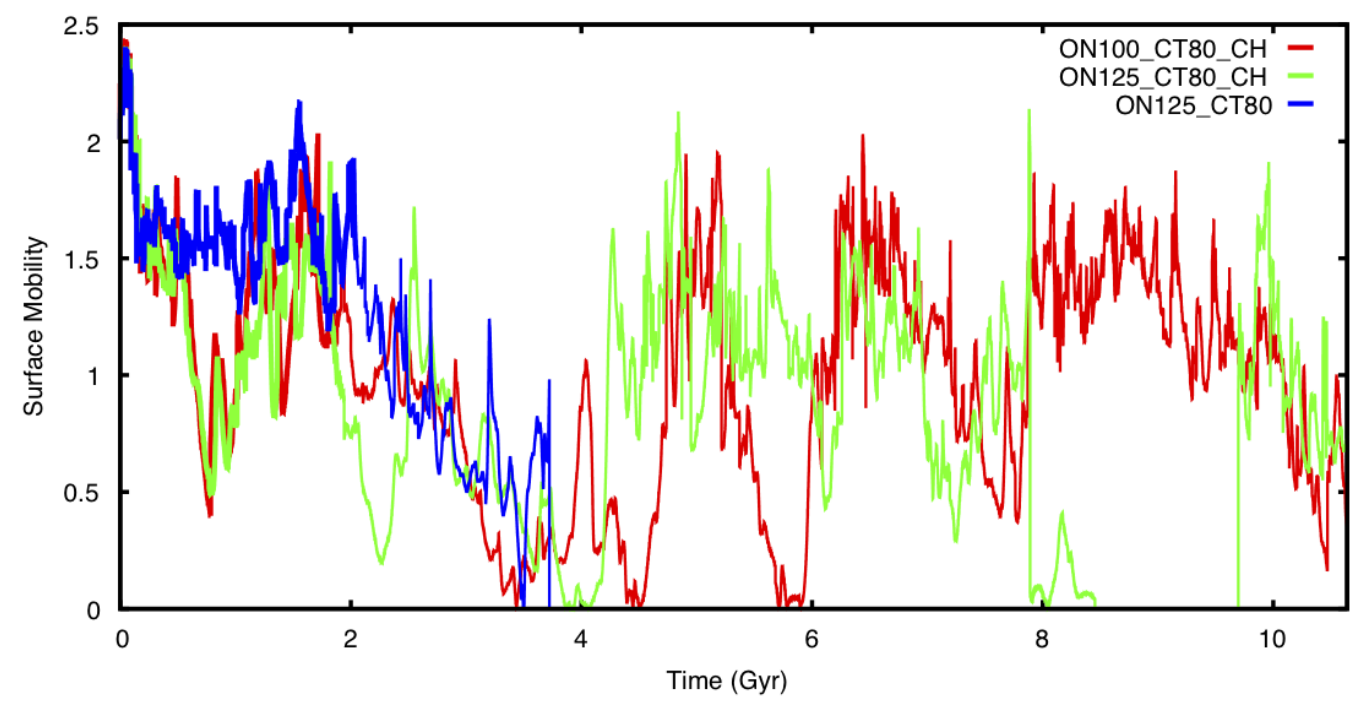

Figure 10. Time series of surface mobility for Models ON100_CT80_CH, ON125_CT80_CH and ON125_CT80. 

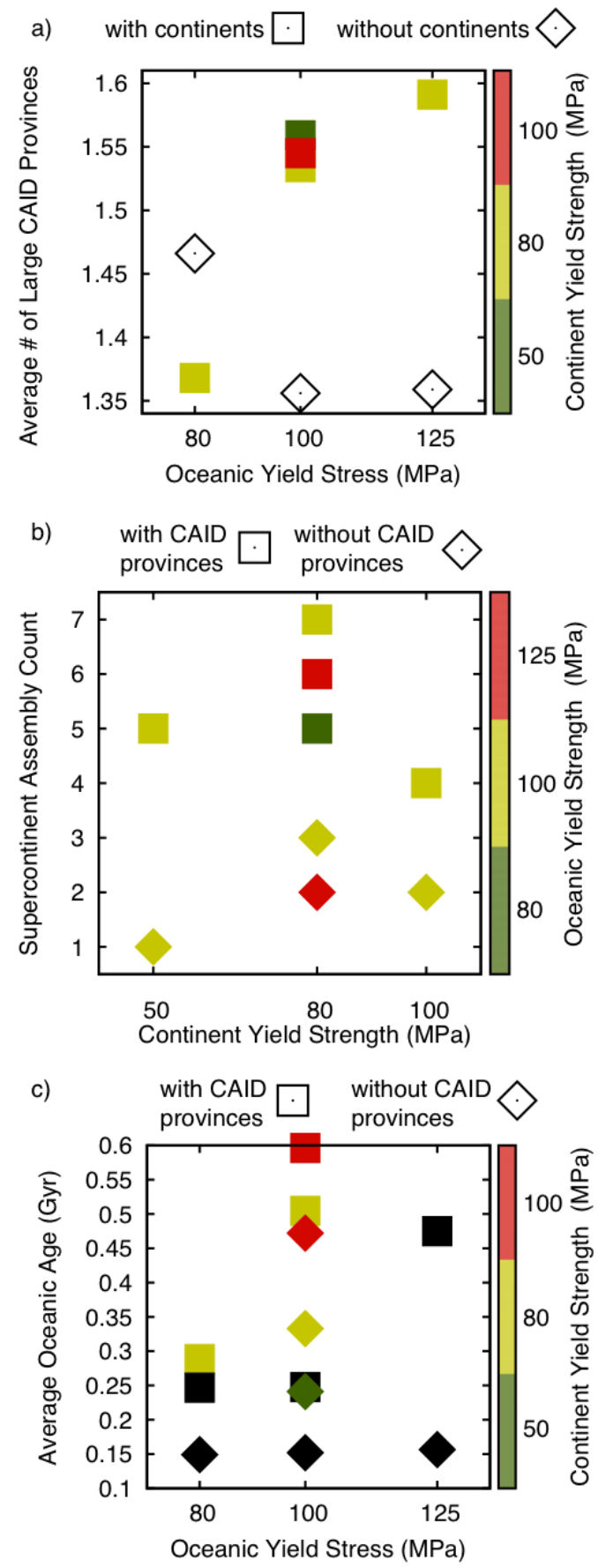

Figure 11. Plots of a) the time-averaged number of large compositional provinces, b) the number of supercontinent aggregations, and c) the average oceanic age, in oceanic/continental tensile yield strength parameter space. Black symbols in part $\mathrm{c}$ do not feature continents. 


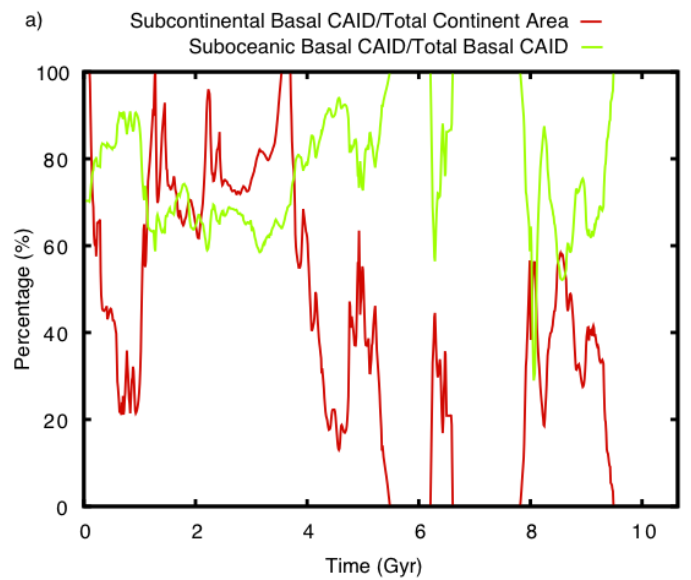

b) Subcontinental Basal CAID/Total Continent Area
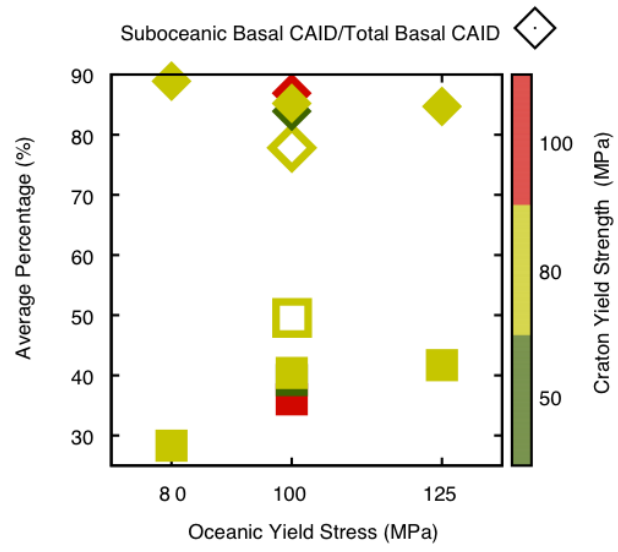

Figure 12. Plots of a) time series of subcontinental and suboceanic basal CAID correlation for Model ON100_CT80_CH and b) time-averaged correlation statistics in oceanic/continental yield strength parameter space. Closed symbols correspond to the models that utilize Initial Condition 1 (Fig. 1). Open symbols correspond to the case started from Initial Condition 2 (Fig. 1). 


\section{A: Resolution test}

In order to evaluate the resolution used for the models listed in Table 1, we have performed several test calculations featuring varied grid and tracer resolutions with identical yield strength values and initial conditions to Model ON100_CT80_CH. The resolution parameters of the test calculations are summarized in Table A.1 using the naming syntax NZm_TPC $n\left\{{ }_{-} \mathrm{RK} 4\right\}$ where $m$ is the number of grid cells in the vertical direction of the uniform mesh and $n$ is the number of tracers per cell at $t=0$. In addition, a test calculation was performed using a fourth order Runge-Kutta scheme to advect the tracers and is indicated using the suffix RK4. In calculations featuring increased grid resolution, linear interpolation was used on the surface age and temperature fields in Fig. 1 to obtain the initial conditions at higher resolution (the initial composition field is defined analytically and does not require interpolation).

Figure A.1 shows time series of the volume average of CAID material for the test calculations outlined in Table A.1. As a reference, we show calculation NZ400_TPC16 (green curve) which is identical to Model ON100_CT80_CH from Table 1. Mass loss for this calculation remains less than 2.8\%. Calculation NZ400_TPC25 (red curve) features an increase in the number of tracers used and results in a mass loss of less than 2.4\%. Calculation NZ450_TPC12 (dark blue curve) features approximately the same number of tracers as calculation NZ400_TPC16 but at increased grid resolution. This calculation results in a maximum mass loss of $2.4 \%$. Calculation NZ450_TPC16 features an increased grid resolution and tracer count compared to calculation NZ400_TPC16 and results in a maximum mass loss of 2.3\%. We find that increasing either the tracer count or the grid resolution reduces the mass error. However, the improvement in mass conservation experiences diminishing returns. For instance, calculation NZ450_TPC16 results in a $0.5 \%$ maximum mass error improvement over calculation NZ400_TPC16 with a 63\% increase in computational cost (see Table A.1).

Calculation NZ400_TPC16_RK4 (cyan curve) features a fourth order Runge-Kutta tracer advection scheme and results in a maximum mass loss of $2.5 \%$. This is a slight improvement in mass conservation compared to using a second order Runge-Kutta advection scheme. Within MC3D, the velocities are known to within second order time accuracy which may explain why using a fourth order Runge-Kutta scheme does not result in a more significant improvement.

\section{Acknowledgments}

We thank Qinya Liu for generous access to computer resources. S.J.T. and J.P.L. are grateful for funding from the NSERC of Canada (fund: 327084-10). Calculations were performed 
on the GPC and Sandy supercomputers at the SciNet HPC Consortium. SciNet is funded by: the Canada Foundation for Innovation under the auspices of Compute Canada; the Government of Ontario; Ontario Research Fund - Research Excellence; and the University of Toronto. Data supporting this study is available from S.J.T upon request.

\section{References}

Allègre, C. J., T. Staudacher, and P. Sarda (1987), Rare gas systematics: formation of the atmosphere, evolution and structure of the Earth's mantle, Earth Planet. Sci. Lett., 81(2), 127-150, doi:10.1016/0012-821X(87)90151-8.

Austermann, J., B. T. Kaye, J. X. Mitrovica, and P. Huybers (2014), A statistical analysis of the correlation between large igneous provinces and lower mantle seismic structure, Geophys. J. Int., 197(1), 1-9, doi:10.1093/gji/ggt500.

Boyet, M., and R. W. Carlson (2006), A new geochemical model for the Earth's mantle inferred from 146 sm-142 nd systematics, Earth Planet. Sci. Lett., 250(1), 254-268, doi:10.1016/j.epsl.2006.07.046.

Brandenburg, J., E. H. Hauri, P. E. van Keken, and C. J. Ballentine (2008), A multiplesystem study of the geochemical evolution of the mantle with force-balanced plates and thermochemical effects, Earth Planet. Sci. Lett., 276(1), 1-13, doi: 10.1016/j.eps1.2008.08.027.

Brandenburg, J. P., and P. E. van Keken (2007), Deep storage of oceanic crust in a vigorously convecting mantle, J. Geophys. Res., 112, B06,403, doi:10.1029/2007GC001692.

Brodholt, J. P., G. Helffrich, and J. Trampert (2007), Chemical versus thermal heterogeneity in the lower mantle: The most likely role of anelasticity, Earth Planet. Sci. Lett., 262(3-4), 429 - 437, doi:10.1016/j.eps1.2007.07.054.

Burke, K., B. Steinberger, T. H. Torsvik, and M. A. Smethurst (2008), Plume generation zones at the margins of large low shear velocity provinces on the core-mantle boundary, Earth Planet. Sci. Lett., 265, 49-60, doi:10.1016/j.epsl.2007.09.042.

Condie, K. C. (2002), Breakup of a paleoproterozoic supercontinent, Gondwana Res., 5(1), 41-43, doi:10.1016/S1342-937X(05)70886-8.

Davaille, A. (1999), Two-layer thermal convection in miscible viscous fluids, J. Fluid Mech., 379, 223-253, doi:10.1017/S0022112098003322.

Davies, D., S. Goes, and H. Lau (2015), Thermally dominated deep mantle llsvps: a review, in The Earth's Heterogeneous Mantle, pp. 441-477, Springer, doi:10.1007/978-3- 
319-15627-9_14.

Davies, G. F. (1977), Whole-mantle convection and plate tectonics, Geophys. J. Int., 49(2), 459-486, doi:10.1111/j.1365-246X.1977.tb03717.x.

Deschamps, F., and P. J. Tackley (2009), Searching for models of thermo-chemical convection that explain probabilistic tomography. ii-influence of physical and compositional parameters, Phys. Earth Planet. Int., 176, 1-18, doi:10.1016/j.pepi.2009.03.012.

Deschamps, F., E. Kaminski, and P. J. Tackley (2011), A deep mantle origin for the primitive signature of ocean island basalt, Nature Geosci., 4(12), 879-882, doi: 10.1038/ngeo1295.

Dziewonski, A. M., V. Lekic, and B. A. Romanowicz (2010), Mantle anchor structure: an argument for bottom up tectonics, Earth Planet. Sci. Lett., 299(1), 69-79, doi: 10.1016/j.epsl.2010.08.013.

Gable, C. W., R. J. O'Connell, and B. J. Travis (1991), Convection in three dimensions with surface plates: Generation of toroidal flow, J. Geophys. Res., 96, 8391-8405, doi: 10.1029/90JB02743.

Garnero, E. J., and A. K. McNamara (2008), Structure and dynamics of Earth's lower mantle, Science, 320, 626-628, doi:10.1126/science.1148028.

Gurnis, M. (1988), Large-scale mantle convection and the aggregation and dispersal of supercontinents, Nature, 332(6166), 695-699, doi:10.1038/332695a0.

Hager, B. H. (1984), Subducted slabs and the geoid: Constraints on mantle rheology and flow, J. Geophys. Res.- Sol. Ea., 89(B7), 6003-6015, doi:10.1029/JB089iB07p06003.

Hager, B. H., and R. J. O’Connell (1981), A simple global model of plate dynamics and mantle convection, J. Geophys. Res.- Sol. Ea., 86(B6), 4843-4867, doi: 10.1029/JB086iB06p04843.

Hernlund, J. W., and C. Houser (2008), On the statistical distribution of seismic velocities in Earth's deep mantle, Earth Planet. Sci. Lett., 265(3), 423-437, doi: 10.1016/j.eps1.2007.10.042.

Heron, P. J., and J. P. Lowman (2011), The effects of supercontinent size and thermal insulation on the formation of mantle plumes, Tectonophysics, 510(1), 28-38, doi: 10.1016/j.tecto.2011.07.002.

Heron, P. J., J. P. Lowman, and C. Stein (2015), Influences on the positioning of mantle plumes following supercontinent formation, J. Geophys. Res.- Sol. Ea., 120(5), 36283648, doi:10.1002/2014JB011727. 
Humayun, M., L. Qin, and M. D. Norman (2004), Geochemical evidence for excess iron in the mantle beneath Hawaii, Science, 306, 91-94, doi:10.1126/science.1101050.

Ishii, M., and J. Tromp (1999), Normal-mode and free-air gravity constraints on lateral variations in velocity and density of Earth's mantle, Science, 285, 1231-1236, doi: 10.1126/science.285.5431.1231.

Ishii, M., and J. Tromp (2004), Constraining large-scale mantle heterogeneity using mantle and inner-core sensitive normal modes, Phys. Earth Planet. Int., 146(1), 113-124, doi:10.1016/j.pepi.2003.06.012.

Jackson, M. G., R. W. Carlson, M. D. Kurz, P. D. Kempton, D. Francis, and J. Blusztajn (2010), Evidence for the survival of the oldest terrestrial mantle reservoir, Nature, 466(7308), 853-856, doi:10.1038/nature09287.

Jarvis, G. T., and D. P. McKenzie (1980), Convection in a compressible fluid with infinite prandtl number, J. Fluid Mech., 96(03), 515-583.

Jellinek, A. M., and M. Manga (2002), The influence of a chemical boundary layer on the fixity, spacing and lifetime of mantle plumes, Nature, 418(6899), 760-763.

Karato, S., and B. B. Karki (2001), Origin of lateral variation of seismic wave velocities and density in the deep mantle, J. Geophys. Res.- Sol. Ea. (1978-2012), 106(B10), 21,771-21,783, doi:10.1029/2001JB000214.

King, S. D., and G. Masters (1992), An inversion for radial viscosity structure using seismic tomography, Geophys. Res. Lett., 19(15), 1551-1554, doi:10.1029/92GL01700.

King, S. D., J. P. Lowman, and C. W. Gable (2002), Episodic tectonic plate reorganizations driven by mantle convection, Earth Planet. Sci. Lett., 203(1), 83-91, doi: 10.1016/S0012-821X(02)00852-X.

Koglin, D. E., S. R. Ghias, S. D. King, G. T. Jarvis, and J. P. Lowman (2005), Mantle convection with reversing mobile plates: A benchmark study, Geochem. Geophys. Geosys., 6, Q09,003, doi:10.1029/2005GC000924.

Kumar, P., X. Yuan, M. R. Kumar, R. Kind, X. Li, and R. Chadha (2007), The rapid drift of the Indian tectonic plate, Nature, 449(7164), 894-897, doi:10.1038/nature06214.

Labrosse, S., J. Hernlund, and N. Coltice (2007), A crystallizing dense magma ocean at the base of the Earth's mantle, Nature, 450, 866-869, doi:10.1038/nature06355.

Le Bars, M., and A. Davaille (2002), Stability of thermal convection in two superimposed miscible viscous fluids, J. Fluid Mech., 471, 339-363, doi:10.1017/S0022112002001878. 
Le Bars, M., and A. Davaille (2004a), Large interface deformation in two-layer thermal convection of miscible viscous fluids, J. Fluid Mech., 499, 75-110, doi: $10.1017 / \mathrm{S} 0022112003006931$.

Le Bars, M., and A. Davaille (2004b), Whole layer convection in a heterogeneous planetary mantle, J. Geophys. Res.- Sol. Ea., 109(B3), doi:10.1029/2003JB002617.

Lekic, V., S. Cottaar, A. Dziewonski, and B. Romanowicz (2012), Cluster analysis of global lower mantle tomography: A new class of structure and implications for chemical heterogeneity, Earth Planet. Sci. Lett., 357, 68-77, doi:10.1016/j.epsl.2012.09.014.

Lenardic, A., L. Moresi, A. Jellinek, C. O’Neill, C. Cooper, and C. Lee (2011), Continents, supercontinents, mantle thermal mixing, and mantle thermal isolation: Theory, numerical simulations, and laboratory experiments, Geochem. Geophys. Geosys., 12(10), doi:10.1029/2011GC003663.

Li, M., and A. K. McNamara (2013), The difficulty for subducted oceanic crust to accumulate at the Earth's core-mantle boundary, J. Geophys. Res., 118, 1807-1816, doi: 10.1002/jgrb.50156.

Li, Y., F. Deschamps, and P. J. Tackley (2014), The stability and structure of primordial reservoirs in the lower mantle: insights from models of thermochemical convection in three-dimensional spherical geometry, Geophys. J. Int., 199(2), 914-930, doi:10.1093/gji/ggu295.

Li, Z.-X., and S. Zhong (2009), Supercontinent-superplume coupling, true polar wander and plume mobility: plate dominance in whole-mantle tectonics, Phys. Earth Planet. Int., 176(3), 143-156, doi:10.1016/j.pepi.2009.05.004.

Long, M. D., and T. W. Becker (2010), Mantle dynamics and seismic anisotropy, Earth Planet. Sci. Lett., 297(3), 341-354, doi:10.1016/j.eps1.2010.06.036.

Lowman, J. P., and C. W. Gable (1999), Thermal evolution of the mantle following continental aggregation in 3D convection models, Geophys. Res. Lett., 26(17), 2649-2652, doi:10.1029/1999GL008332.

Lowman, J. P., S. D. King, and C. W. Gable (2003), The role of the heating mode of the mantle in intermittent reorganization of the plate velocity field, Geophys. J. Int., 152(2), 455-467, doi:10.1046/j.1365-246X.2003.01862.x.

Masters, G., G. Laske, H. Bolton, and A. Dziewonski (2000), The relative behavior of shear velocity, bulk sound speed, and compressional velocity in the mantle: Implications for chemical and thermal structure, Earth's deep interior: mineral physics and 
tomography from the atomic to the global scale, pp. 63-87, doi:10.1029/GM117p0063.

McNamara, A. K., and S. Zhong (2004a), Thermochemical structures within a spherical mantle: Superplumes or piles?, J. Geophys. Res.- Sol. Ea., 109(B7), doi: 10.1029/2003JB002847.

McNamara, A. K., and S. Zhong (2004b), The influence of thermochemical convection on the fixity of mantle plumes, Earth Planet. Sci. Lett., 222(2), 485-500, doi: http://dx.doi.org/10.1016/j.epsl.2004.03.008.

McNamara, A. K., and S. Zhong (2005), Thermochemical structures beneath Africa and the Pacific ocean, Nature, 437, 1136-1139, doi:10.1038/nature04066.

Mitchell, R. N., W. Bleeker, O. Van Breemen, T. N. Lecheminant, P. Peng, M. K. Nilsson, and D. A. Evans (2014), Plate tectonics before 2.0 Ga: Evidence from paleomagnetism of cratons within supercontinent Nuna, Am. J. Sci., 314(4), 878-894, doi: 10.2475/04.2014.03.

Mitrovica, J. X., and A. M. Forte (1997), Radial profile of mantle viscosity: results from the joint inversion of convection and postglacial rebound observables, J. Geophys. Res.Sol. Ea., 102(B2), 2751-2769, doi:10.1029/96JB03175.

Monnereau, M., and S. Quéré (2001), Spherical shell models of mantle convection with tectonic plates, Earth Planet. Sci. Lett., 184(3), 575-587, doi:10.1016/S0012821X(00)00334-4.

Montague, N. L., and L. H. Kellogg (2000), Numerical models of a dense layer at the base of the mantle and implications for the geodynamics of D”, J. Geophys. Res., 105, 11,101-11,114, doi:10.1029/1999JB900450.

Moresi, L., and V. Solomatov (1998), Mantle convection with a brittle lithosphere: thoughts on the global tectonic styles of the Earth and Venus, Geophys. J. Int., 133(3), 669-682, doi:10.1046/j.1365-246X.1998.00521.x.

Murakami, M., K. Hirose, K. Kawamura, N. Sata, and Y. Ohishi (2004), Postperovskite phase transition in MgSiO3, Science, 304(5672), 855-858, doi: 10.1126/science.1095932.

Ni, S., E. Tan, M. Gurnis, and D. Helmberger (2002), Sharp sides to the African superplume, Science, 296(5574), 1850-1852, doi:10.1126/science.1070698.

O'Farrell, K. A., and J. P. Lowman (2010), Emulating the thermal structure of spherical shell convection in plane-layer geometry mantle convection models, Phys. Earth Planet. Int., 182, 73-84, doi:10.1016/j.pepi.2010.06.010. 
O'Farrell, K. A., J. P. Lowman, and H.-P. Bunge (2013), Comparison of spherical-shell and plane-layer mantle convection thermal structure in viscously stratified models with mixed-mode heating: implications for the incorporation of temperature-dependent parameters, Geophys. J. Int., 192, 456-472, doi:10.1093/gji/ggs053.

O'Neill, C., A. Lenardic, A. Jellinek, and L. Moresi (2009), Influence of supercontinents on deep mantle flow, Gondwana Res., 15(3), 276-287, doi:10.1016/j.gr.2008.11.005.

Paulson, A., S. Zhong, and J. Wahr (2007), Inference of mantle viscosity from grace and relative sea level data, Geophys. J. Int., 171(2), 497-508, doi:10.1111/j.1365246X.2007.03556.x.

Ritsema, J., S. Ni, D. V. Helmberger, and H. P. Crotwell (1998), Evidence for strong shear velocity reductions and velocity gradients in the lower mantle beneath Africa, Geophys. Res. Lett., 25(23), 4245-4248, doi:10.1029/1998GL900127.

Rolf, T., N. Coltice, and P. Tackley (2012), Linking continental drift, plate tectonics and the thermal state of the Earth's mantle, Earth Planet. Sci. Lett., 351, 134-146, doi: 10.1016/j.eps1.2012.07.011.

Runcorn, S. K. (2013), Continental drift, vol. 3, Elsevier.

Saltzer, R. L., R. D. van der Hilst, and H. Kárason (2001), Comparing p and s wave heterogeneity in the mantle, Geophys. Res. Lett., 28(7), 1335-1338, doi: 10.1029/2000GL012339.

Samuel, H., and M. Evonuk (2010), Modeling advection in geophysical flows with particle level sets, Geochem. Geophys. Geosys., 11(8), doi:10.1029/2010GC003081.

Schuberth, B. S. A., H.-P. Bunge, and J. Ritsema (2009), Tomographic filtering of highresolution mantle circulation models: Can seismic heterogeneity be explained by temperature alone?, Geochem. Geophys. Geosys., 10(5), doi:10.1029/2009GC002401.

Schuberth, B. S. A., C. Zaroli, and G. Nolet (2012), Synthetic seismograms for a synthetic Earth: long-period p- and s-wave traveltime variations can be explained by temperature alone, Geophys. J. Int., 188(3), 1393-1412, doi:10.1111/j.1365-246X.2011.05333.x.

Scotese, C. R., and W. McKerrow (1991), Ordovician plate tectonic reconstructions, Geolog. Surv. Can. Pap., 90(9), 271-282.

Shahnas, M. H., and G. T. Jarvis (2007), On the relative importance of mineral phase transitions and viscosity stratification in controlling the sinking rates of detached slab remnants, Geophys. Res. Lett., 34(11), doi:10.1029/2007GL029463. 
Šrámek, O., W. F. McDonough, E. S. Kite, V. Lekić, S. T. Dye, and S. Zhong (2013), Geophysical and geochemical constraints on geoneutrino fluxes from Earth's mantle, Earth Planet. Sci. Lett., 361, 356-366, doi:10.1016/j.eps1.2012.11.001.

Stein, C., and U. Hansen (2014), Numerical investigation of a layered temperaturedependent viscosity convection in comparison to convection with a full temperature dependence, Phys. Earth Planet. Int., 226, 1-13, doi:10.1016/j.pepi.2013.11.004.

Stein, C., J. Lowman, and U. Hansen (2013), The influence of mantle internal heating on lithospheric mobility: Implications for super-Earths, Earth Planet. Sci. Lett., 361, 448-459, doi:10.1016/j.eps1.2012.11.011.

Stein, C., J. Lowman, and U. Hansen (2014), A comparison of mantle convection models featuring plates, Geochem. Geophys. Geosys., 15, doi:10.1002/2013GC005211.

Styles, E., D. R. Davies, and S. Goes (2011), Mapping spherical seismic into physical structure: biases from 3-d phase-transition and thermal boundary-layer heterogeneity, Geophys. J. Int., 184(3), 1371-1378, doi:10.1111/j.1365-246X.2010.04914.x.

Tackley, P. J. (2000), Self-consistent generation of tectonic plates in time-dependent, three-dimensional mantle convection simulations, Geochem. Geophys. Geosys., 1, 1021, doi:10.1029/2000GC000036.

Tackley, P. J. (2012), Dynamics and evolution of the deep mantle resulting from thermal, chemical, phase and melting effects, Earth-Sci. Rev., 110(1), 1-25, doi: 10.1016/j.earscirev.2011.10.001.

Tackley, P. J., and S. D. King (2003), Testing the tracer ratio method for modeling active compositional fields in mantle convection simulations, Geochem. Geophys. Geosys., 4, 4, doi:10.1029/2001GC000214.

Tackley, P. J., S. Xie, T. Nakagawa, and J. W. Hernlund (2005), Numerical and laboratory studies of mantle convection: Philosophy, accomplishments, and thermochemical structure and evolution, Earth's Deep Mantle: Structure, Composition, and Evolution, 160, 83-99, doi:10.1029/160GM07.

Tan, E., W. Leng, S. Zhong, and M. Gurnis (2011), On the location of plumes and lateral movement of thermochemical structures with high bulk modulus in the 3-D compressible mantle, Geochem. Geophys. Geosys., 12, Q07,005, doi:10.1029/2011GC003665.

To, A., B. Romanowicz, Y. Capdeville, and N. Takeuchi (2005), 3D effects of sharp boundaries at the borders of the African and Pacific superplumes: Observation and modeling, Earth Planet. Sci. Lett., 233(1-2), 137 - 153, doi:10.1016/j.epsl.2005.01.037. 
Torsvik, T. H., M. A. Smethurst, K. Burke, and B. Steinberger (2006), Large igneous provinces generated from the margins of the large low-velocity provinces in the deep mantle, Geophys. J. Int., 167(3), 1447-1460, doi:10.1111/j.1365-246X.2006.03158.x.

Torsvik, T. H., K. Burke, B. Steinberger, S. J. Webb, and L. D. Ashwal (2010), Diamonds sampled by plumes from the core-mantle boundary, Nature, 466, 352-355, doi:10.1038/nature09216.

Trampert, J., F. Deschamps, J. Resovsky, and D. Yuen (2004), Probabilistic tomography maps chemical heterogeneities throughout the lower mantle, Science, 306(5697), 853-856, doi:10.1126/science.1101996.

Trim, S., P. Heron, C. Stein, and J. Lowman (2014), The feedback between surface mobility and mantle compositional heterogeneity: Implications for the Earth and other terrestrial planets, Earth Planet. Sci. Lett., 405, 1-14, doi:10.1016/j.epsl.2014.08.019. van Heck, H. J., and P. J. Tackley (2011), Plate tectonics on super-Earths: Equally or more likely than on Earth, Earth Planet. Sci. Lett., 310, 252-261, doi: 10.1016/j.eps1.2011.07.029.

van Keken, P., S. King, H. Schmeling, U. Christensen, D. Neumeister, and M.-P. Doin (1997), A comparison of methods for the modeling of thermochemical convection, $J$. Geophys. Res.- Sol. Ea., 102(B10), 22,477-22,495, doi:10.1029/97JB01353.

Weller, M. B., and A. Lenardic (2012), Hysteresis in mantle convection: Plate tectonics systems, Geophys. Res. Lett., 39, L10,202, doi:10.1029/2012GL051232.

Wilson, J. T. (1966), Did the Atlantic close and then re-open?, Nature, 211, 676-681, doi:10.1038/211676a0.

Wookey, J., S. Stackhouse, J.-M. Kendall, J. Brodholt, and G. D. Price (2005), Efficacy of the post-perovskite phase as an explanation for lowermost-mantle seismic properties, Nature, 438(7070), 1004-1007, doi:10.1038/nature04345.

Zhang, N., S. Zhong, W. Leng, and Z.-X. Li (2010), A model for the evolution of the Earth's mantle structure since the early paleozoic, J. Geophys. Res.- Sol. Ea., 115(B6), doi:10.1029/2009JB006896. 
Table A.1. Resolution Test Calculation Parameters ${ }^{a}$

\begin{tabular}{lcccc}
\hline Calculation Name & Grid Resolution & Tracers per Cell at $t=0$ & Number of Tracers & Computation Time (Hours) \\
\hline NZ400_TPC16 & $4000 \times 400$ & 16 & $2.56 \times 10^{7}$ & 111 \\
NZ400_TPC25 & $4000 \times 400$ & 25 & $4 \times 10^{7}$ & 128 \\
NZ450_TPC12 & $4500 \times 450$ & 12 & $2.43 \times 10^{7}$ & 176 \\
NZ450_TPC16 & $4500 \times 450$ & 16 & $3.24 \times 10^{7}$ & 181 \\
NZ400_TPC16_RK4 & $4000 \times 400$ & 16 & $2.56 \times 10^{7}$ & 137 \\
\hline
\end{tabular}

${ }^{a}$ Summary of resolution parameters and computation time for test calculations featuring identical yield strength values and initial conditions to Model ON100_CT80_CH. Calculations were performed using six octal core Intel Xeon E5-2650 2.6GHz CPUs (a total of 48 cores). Calculation names are described in the text. 


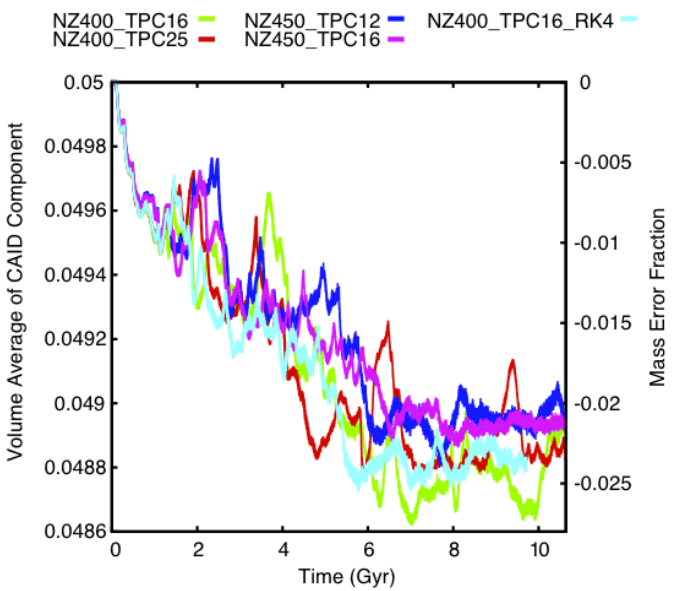

Figure A.1. Time series of the volume average of CAID material in test calculations featuring increased grid and tracer resolutions with identical yield strength parameters and initial conditions to Model ON100_CT80_CH. Calculation names (described in the text) are indicated in the legend. 